\title{
Projecting an extended formulation for mixed integer covers on bipartite graphs
}

\author{
Michele Conforti* Laurence A. Wolsey ${ }^{\dagger} \quad$ Giacomo Zambelli*
}

November 18, 2008, Revised November 2009

\begin{abstract}
We consider the mixed integer version of bipartite vertex cover. This is equivalent to the mixed integer network dual model, recently introduced in [2], that generalizes several mixed integer sets arising in production planning.

We derive properties of inequalities that are valid for the convex hull of the mixed integer bipartite covers by projecting an extended formulation onto the space of the original variables. This permits us to give a complete description of the facet inducing inequalities of the double mixing set and of the continuous mixing set with flows, two mixed integer sets that generalize several models studied in the literature.
\end{abstract}

\section{Introduction}

Given a bipartite graph $G=(U \cup V, E)$ a set $I \subseteq U \cup V$ and rational numbers $b_{i j}, i j \in E$, we study the set of mixed integer vertex covers

$$
S^{(G, I)}=\left\{x \in \mathbb{R}^{U \cup V} \mid x_{i}+x_{j} \geq b_{i j} \quad i j \in E ; x_{i} \in \mathbb{Z} \quad i \in I\right\} .
$$

We show that the set $S^{(G, I)}$ is equivalent to the "network dual" set introduced and studied recently by Conforti, Di Summa, Eisenbrand and Wolsey [2] as a generalization of several mixed integer sets that have appeared in the literature $[3,5,10,13]$.

As an example of a mixed integer set that can be transformed into the form $S^{(G, I)}$, we mention the continuous mixing set, studied by Van Vyve [13], defined as $X^{C M}=\left\{(s, r, x) \in \mathbb{R} \times \mathbb{R}_{+}^{n} \times \mathbb{Z}^{n} \mid s+r_{i}+x_{i} \geq b_{i}, i=1, \ldots, n\right\}$.

\footnotetext{
* Dipartimento di Matematica Pura ed Applicata, Università degli Studi di Padova. Via Trieste 63, 35121 Padova, Italy (conforti@math.unipd.it, giacomo@math.unipd.it).

${ }^{\dagger}$ Center of Operations Research and Econometrics (CORE), Université catholique de Louvain. 34 Voie du Roman Pays, 1348 Louvain-la-Neuve, Belgium (laurence.wolsey@uclouvain.be)
} 
Introducing new variables $\tau=-s, y_{i}=s+r_{i}$ and substituting for $s$ and $r_{i}$, the set $X^{C M}$ is transformed into the set $\left\{(\tau, y, x) \in \mathbb{R} \times \mathbb{R}^{n} \times \mathbb{Z}^{n} \mid \tau+y_{i} \geq\right.$ $\left.0, y_{i}+x_{i} \geq b_{i}, i=1, \ldots, n\right\}$, where the inequality $r_{i} \geq 0$ becomes $\tau+y_{i} \geq 0$. The above set is of the form $S^{(G, I)}$, where $G$ is a tree on the node set $\left\{\tau, y_{i}, x_{i}, i=1, \ldots, n\right\}$ and edge set $\left\{\tau y_{i}, y_{i} x_{i}, i=1, \ldots, n\right\}$.

An extended formulation of a polyhedron $P$ in the $x$-space is an inequality description $A x+B \mu \geq d$ of a polyhedron $Q$ in the $(x, \mu)$-space such that $P$ is the projection of $Q$ in the $x$-space.

Conforti et al. [2] give an extended formulation for $\operatorname{conv}\left(S^{(G, I)}\right)$, whose size, however, is not always polynomial in $|E|$ and the size of $b_{e}, e \in E$. In that paper, the authors show that for many models of the type $S^{(G, I)}$ arising in the literature, the extended formulation has polynomial size. Hence, in those cases, the problem of optimizing a linear function over $S^{(G, I)}$ is polynomial time. However in general the computational complexity of optimizing a linear function over $S^{(G, I)}$ is not known.

An inequality description of $\operatorname{conv}\left(S^{(G, I)}\right)$, in the original space of the $x$ variables is not known in general, except for the case where the $b_{e} \mathrm{~s}$ are half-integral [4] or for specific graph topologies. To find such a description, one possible approach is to try to characterize the facet-defining inequalities of $\operatorname{conv}\left(S^{(G, I)}\right)$ by projection of an extended formulation, and this is the subject of this paper.

In Section 2 we derive an extended formulation for $\operatorname{conv}\left(S^{(G, I)}\right)$. This formulation differs slightly from that proposed in [2], but its projection cone is easier to analyze. Throughout the paper, $k$ denotes the smallest integer such that $k b_{i j} \in \mathbb{Z}$ for all $i j \in E$. The extended formulation we describe is polynomial in $|E|$ and $k$. Section 3 is devoted to showing the equivalence of the "network dual" set of [2] and the set $S^{(G, I)}$.

In Section 4, we give several properties of the rays of the projection cone of the extended formulation that generate facets of $\operatorname{conv}\left(S^{(G, I)}\right)$. Special emphasis is given to the case in which $G$ is a tree. Many models studied in the literature fit this case $[3,5,10,13]$. In particular, a "cyclic property" of the coefficients of the rays, given in Lemma 19, will prove crucial in deriving facet-defining inequalities of $\operatorname{conv}\left(S^{G, I}\right)$ when $G$ is a tree.

In Section 5 we rely on the results of the previous section to obtain explicit inequality descriptions for some mixed integer sets. More precisely, we describe the convex hull of the double mixing set,

$$
X^{G M I X}=\left\{\left(x_{0}, x\right) \in \mathbb{R} \times \mathbb{Z}^{n} \mid b_{i} \leq x_{0}+x_{i} \leq c_{i}, i=1, \ldots, n\right\},
$$

and of the continuous mixing set with flows

$X^{C M F}=\left\{(s, r, z, x) \in \mathbb{R} \times \mathbb{R}_{+}^{n} \times \mathbb{R}_{+}^{n} \times \mathbb{Z}^{n} \mid s+r_{i}+z_{i} \geq b_{i}, z_{i} \leq x_{i}, i=1, \ldots, n\right\}$.

Both results are new. The characterization of the continuous mixing set generalizes work of Van Vyve [13] and solves an open problem in [3]. 


\section{The extended formulation}

We assume here that $k$ is a positive integer and that $b_{i j}=\left\lfloor b_{i j}\right\rfloor+\frac{h_{i j}}{k}$ for some integer $h_{i j}$ between 0 and $k-1$ for each $i j \in E$,.

Let $L=V(G) \backslash I$. For any vector $x \in \mathbb{R}^{V(G)}$, we denote by $x_{I}$ and $x_{L}$ its restrictions to the components indexed by $I$ and $L$ respectively.

Remark 1 Every point of $S^{(G, I)}$ is a convex combination of points $x^{1}, \ldots, x^{h} \in$ $S^{(G, I)}$ such that $k x^{\ell}$ is integral for $\ell=1, \ldots, h$.

Proof: The constraint matrix of the system $x_{i}+x_{j} \geq b_{i j}, i j \in E$ is the edgenode incidence matrix $A$ of $G$. Since $G$ is bipartite, $A$ is totally unimodular. Let $\bar{x} \in S(G, I)$. By definition of $S^{(G, I)}, \bar{x}_{I}$ is integral. Furthermore, $\bar{z}=$ $k \bar{x}_{L}$ is in the polyhedron $Q$ defined by $A_{L} z \geq k\left(b-A_{I} \bar{x}_{I}\right)$, where $A_{L}$ and $A_{I}$ are the column submatrices of $A$ indexed by $L$ and $I$, respectively. Since $A_{L}$ is totally unimodular and $k\left(b-A_{I} \bar{x}_{I}\right)$ is an integral vector, $Q$ is an integral polyhedron. Hence $\bar{z}$ is a convex combination of integral points $z^{1}, \ldots, z^{h}$ of $Q$. Let $x^{i}=\left(\bar{x}_{I}, k^{-1} z^{i}\right) \in S^{(G, I)}$, then $\bar{x}$ is a convex combination of $x^{1}, \ldots, x^{h}$.

Remark 2 The polyhedron $\operatorname{conv}\left(S^{(G, I)}\right)$ is not pointed. Indeed the dimension of its lineality space is the number of connected components of $G$. A basis of the lineality space is given by the vectors, defined by each component $C$, of the form $x_{i}=1, i \in U \cap C, x_{i}=-1, i \in V \cap C, x_{i}=0, i \notin C$.

Remark 1 shows that every minimal face of $\operatorname{conv}(S(G, I))$ contains a point that is $1 / k$-integral.

Consider a point $\bar{x}$ of $\operatorname{conv}\left(S^{(G, I)}\right)$ with $k \bar{x}$ is integral. Thus $\bar{x}_{i}=\left\lfloor\bar{x}_{i}\right\rfloor+\frac{r_{i}}{k}$ for some integer $r_{i}, i \in U \cup V$. For every $i \in U \cup V$, define $\bar{\mu}_{i}^{t}=\left\lfloor\bar{x}_{i}\right\rfloor$ for $t=0, \ldots, k-r_{i}-1, \bar{\mu}_{i}^{t}=\left\lceil\bar{x}_{i}\right\rceil$ for $t=k-r_{i}, \ldots, k-1$. Then $\bar{x}_{i}=$ $\frac{1}{k}\left(\bar{\mu}_{i}^{0}+\cdots+\bar{\mu}_{i}^{k-1}\right)$ where $\bar{\mu}_{i}^{0}, \ldots, \bar{\mu}_{i}^{k-1} \in \mathbb{Z}$. Furthermore, if $i \in I$, then $\bar{x}_{i}$ is integer and $\bar{\mu}_{i}^{0}=\ldots=\bar{\mu}_{i}^{k-1}$.

Therefore we associate $k$ auxiliary integer variables $\mu_{i}^{0}, \ldots, \mu_{i}^{k-1}$ to each variable $x_{i}, i \in V(G)$. This allows us to define $\operatorname{conv}\left(S^{(G, I)}\right)$ as the projection of a polyhedron in the $(x, \mu)$ space, as follows.

Theorem 3 The polyhedron $\operatorname{conv}\left(S^{(G, I)}\right)$ is the projection onto the space of the $x$-variables of the polyhedron $\mathcal{Q}_{I}$ defined as the set of points $(x, \mu)$ satisfying

$$
\begin{array}{rc}
x_{i}-\frac{1}{k}\left(\mu_{i}^{0}+\cdots+\mu_{i}^{k-1}\right)=0 & i \in U \cup V \\
\mu_{i}^{t}+\mu_{j}^{k-h_{i j}-1-t} \geq\left\lfloor b_{i j}\right\rfloor & t=0, \ldots, k-h_{i j}-1 \quad i j \in E \\
\mu_{i}^{t}+\mu_{j}^{2 k-h_{i j}-1-t} \geq\left\lceil b_{i j}\right\rceil & t=k-h_{i j}, \ldots, k-1 \quad i j \in E \\
\mu_{i}^{t-1}-\mu_{i}^{t}=0 & t=1, \ldots, k-1, i \in I .
\end{array}
$$




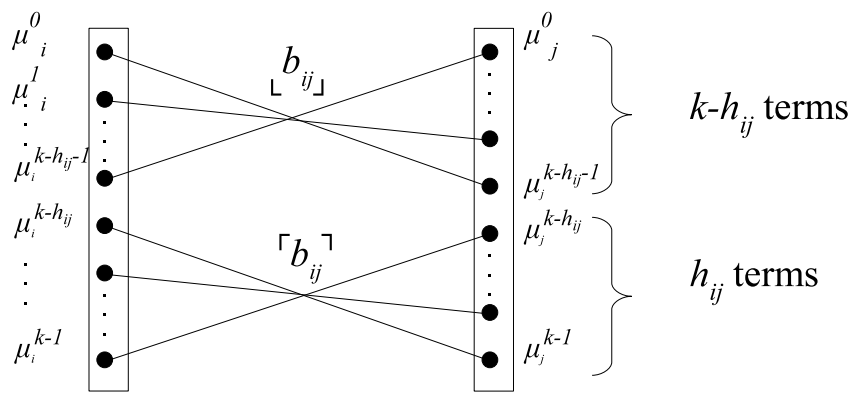

Figure 1: Representation of the extended formulation of a single inequality $x_{i}+x_{j} \geq b_{i j}$. Each edge joins the node corresponding to variable $\mu_{i}^{t}$ on the left to the node corresponding to variable $\mu_{j}^{q_{i j t}}$ on the right. Edges at the top group represent inequalities (2), while edges below represent inequalities (3).

We will need the following result of Heller and Tompkins.

Theorem 4 (Heller and Tompkins [6]) Let $A$ be $a 0, \pm 1$-matrix with at most two nonzero entries per row. The matrix $A$ is totally unimodular if and only if the columns of $A$ can be partitioned into two sets $R$ and $B$ such that the sum of the columns in $R$ minus the sum of the columns in $B$ is a $0, \pm 1$-vector.

For ease of notation, given an edge $i j \in E$ and an index $t, 0 \leq t \leq k-1$, we define

$$
q_{i j t}=\left\{\begin{array}{l}
k-h_{i j}-1-t, \quad t=0, \ldots, k-h_{i j}-1 \\
2 k-h_{i j}-1-t, \quad t=k-h_{i j}, \ldots, k-1
\end{array}\right.
$$

Proof of Theorem 3. We first show that the projection of $\mathcal{Q}_{I}$ onto the $x$ space is contained in $\operatorname{conv}\left(S^{(G, I)}\right)$. Notice that, given ij $\in E$, summing all inequalities (2)-(3) relative to $i j$, dividing them by $k$ and adding the equations $x_{i}-\sum_{t=0}^{k-1} \frac{\mu_{i}^{t}}{k}=0$ and $x_{j}-\sum_{t=0}^{k-1} \frac{\mu_{j}^{t}}{k}=0$, one obtains $x_{i}+x_{j} \geq b_{i j}$. Therefore $x_{i}+x_{j} \geq b_{i j}$ is valid for the projection of $\mathcal{Q}_{I}$.

Let $M$ be the constraint matrix of the system defined by (2),(3),(4). Then $M$ is a $0, \pm 1$ matrix with exactly two nonzero elements in each row, and the sum of the columns of $M$ corresponding to nodes in $U$ minus the sum of the columns corresponding to nodes in $V$ yields the vector of all zeroes. By Theorem 4, matrix $M$ is totally unimodular.

Consider a point $(\bar{x}, \bar{\mu})$ of $\mathcal{Q}_{I}$. We wish to show that $\bar{x} \in \operatorname{conv}\left(S^{(G, I)}\right)$. Since the constraint matrix $M$ of the system defined by $(2),(3),(4)$ is totally unimodular, and the right-hand-side of such system is integral, $\bar{\mu}$ can be written as a convex combination of integral vectors $\mu^{1}, \ldots, \mu^{h}$ satisfying $(2),(3),(4)$. Let $x^{1}, \ldots, x^{h}$ be the vector defined by $\mu^{1}, \ldots, \mu^{h}$ in the 
system of equations (1). By (4), $x_{I}^{1}, \ldots, x_{I}^{h}$ are integral vectors, therefore $x^{1}, \ldots, x^{h} \in S^{(G, I)}$. Furthermore $\bar{x}$ is a convex combination of $x^{1}, \ldots, x^{h}$, thus $\bar{x} \in \operatorname{conv}\left(S^{(G, I)}\right)$.

Conversely, we show that $\operatorname{conv}\left(S^{(G, I)}\right)$ is contained in the projection of $\mathcal{Q}_{I}$ onto the $x$-space. By Remark 1 , given a point $\bar{x}$ of $S^{(G, I)}$ such that $k \bar{x}$ is integral, we only need to show that there is a vector $\bar{\mu}$ such that $(\bar{x}, \bar{\mu}) \in \mathcal{Q}_{I}$. Since $k \bar{x}$ is integral, $\bar{x}_{i}=\left\lfloor\bar{x}_{i}\right\rfloor+\frac{r_{i}}{k}$ for some integer $r_{i}, i \in U \cup V$. Also, $\bar{x}_{i} \in \mathbb{Z}$ for every $i \in I$, thus $r_{i}=0$ for every $i \in I$. For every $i \in U \cup V$, define $\bar{\mu}_{i}^{t}=\left\lfloor\bar{x}_{i}\right\rfloor$ for $t=0, \ldots, k-r_{i}-1, \bar{\mu}_{i}^{t}=\left\lceil\bar{x}_{i}\right\rceil$ for $t=k-r_{i}, \ldots, k-1$. Clearly $(\bar{x}, \bar{\mu})$ satisfies (1) and (4). We now show that $\bar{\mu}$ satisfies (2),(3). In fact, given $i j \in E$ and an index $t, 0 \leq t \leq k-1$, observe that the vector $\bar{\mu}$ defined above satisfies

$$
\begin{aligned}
& \frac{1}{k}\left(\bar{\mu}_{i}^{0}+\ldots+\bar{\mu}_{i}^{k-1}\right)+\frac{1}{k}\left(\bar{\mu}_{j}^{0}+\ldots+\bar{\mu}_{j}^{k-1}\right) \geq b_{i j} ; \\
& \frac{1}{k} \bar{\mu}_{i}^{t}-\frac{1}{k} \bar{\mu}_{i}^{\ell} \geq 0, \quad \ell=0, \ldots, t-1 ; \\
& \frac{1}{k} \bar{\mu}_{i}^{t}-\frac{1}{k} \bar{\mu}_{i}^{\ell} \geq-\frac{1}{k}, \quad \ell=t+1, \ldots, k-1 ; \\
& \frac{1}{k} \bar{\mu}_{j}^{q_{i j t}}-\frac{1}{k} \bar{\mu}_{j}^{\ell} \geq 0, \quad \ell=0, \ldots, q_{i j t}-1 ; \\
& \frac{1}{k} \bar{\mu}_{j}^{q_{i j t}}-\frac{1}{k} \bar{\mu}_{j}^{\ell} \geq-\frac{1}{k}, \quad \ell=q_{i j t}+1, \ldots, k-1 .
\end{aligned}
$$

Summing all these inequalities, we obtain

$$
\bar{\mu}_{i}^{t}+\bar{\mu}_{j}^{q_{i j t}} \geq b_{i j}-\frac{1}{k}\left(2 k-2-t-q_{i j t}\right) .
$$

For $t=0, \ldots, k-h_{i j}-1$, this gives $\bar{\mu}_{i}^{t}+\bar{\mu}_{j}^{q_{i j t}} \geq b_{i j}-1-\frac{1}{k}\left(h_{i j}-1\right)$, that is $\bar{\mu}_{i}^{t}+\bar{\mu}_{j}^{q_{i j t}} \geq\left\lfloor b_{i j}\right\rfloor-1+\frac{1}{k}$. Since $\bar{\mu}$ is integral, it satisfies (2).

For $t=k-h_{i j}, \ldots, k-1$, this gives $\bar{\mu}_{i}^{t}+\bar{\mu}_{j}^{q_{i j t}} \geq b_{i j}-\frac{1}{k}\left(h_{i j}-1\right)$, that is $\bar{\mu}_{i}^{t}+\bar{\mu}_{j}^{q_{i j t}} \geq\left\lfloor b_{i j}\right\rfloor+\frac{1}{k}$. Since $\bar{\mu}$ is integral, it satisfies (3).

Remark 5 Observe that constraints (4) are equivalent to $x_{i}=\mu_{i}^{1}=\ldots=$ $\mu_{i}^{k-1}$ for $i \in I$. Therefore, for $i \in I$, the variables $\mu_{i}^{t}, t=0, \ldots, k-1$ can be eliminated in the system defining $\mathcal{Q}_{I}$ by replacing them with variable $x_{i}$. The constraint matrix of the system obtained from (2),(3), ij $\in E$, by the above substitution is again totally unimodular.

Example. The mixing set [10] is the set

$$
X_{n}^{M I X}=\left\{\left(x_{0}, x\right) \in \mathbb{R} \times \mathbb{Z}^{n} \mid x_{0}+x_{i} \geq b_{i}, i=1, \ldots, n\right\} .
$$

Let $b_{i}=\frac{h_{i}}{k}, i=1, \ldots, n$, where $k$ and $h_{1}, \ldots, h_{n}$ are integer. After replacement of each variable $\mu_{i}^{t}, i=1, \ldots, k, t=0, \ldots, k-1$ by the variable $x_{i}$ as 
explained in Remark 5, the extended formulation for $\operatorname{conv}\left(X_{n}^{M I X}\right)$ given in Theorem 3 becomes

$$
\begin{aligned}
k x_{0}-\mu_{0}^{0}-\cdots-\mu_{0}^{k-1} & =0 \\
\mu_{0}^{t}+x_{i} & \geq\left\lfloor b_{i}\right\rfloor, \quad t=0, \ldots, k-h_{i}-1, i=1, \ldots, n ; \\
\mu_{0}^{t}+x_{i} & \geq\left\lceil b_{i}\right\rceil, \quad t=k-h_{i}, \ldots, k-1, i=1, \ldots, n .
\end{aligned}
$$

Figure 2 depicts the constraints defining $X_{n}^{M I X}$ and the corresponding extended formulation.
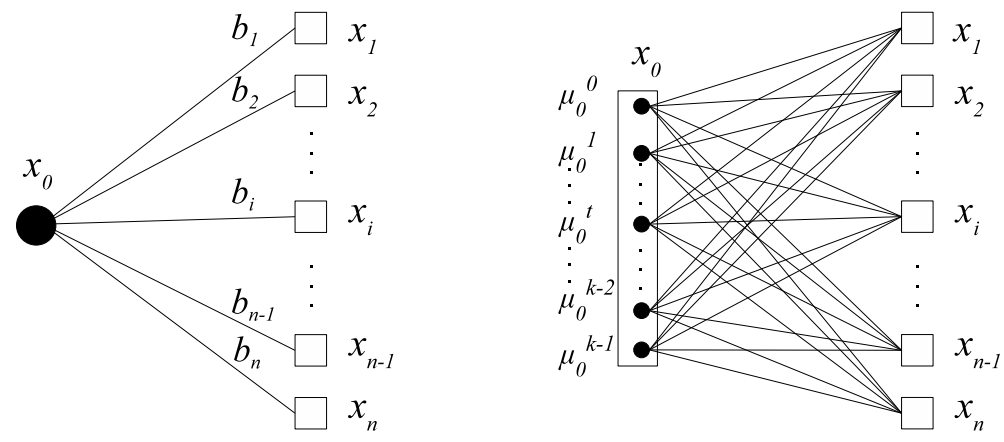

Figure 2: Mixing set and extended formulation.

Remark 6 The extended formulation in Theorem 3 has $O(k|U \cup V|)$ variables and $O(k|E|)$ constraints. Therefore its size is pseudopolynomial, but in general not polynomial, in the size of the encoding of $S^{(G, I)}$.

The extended formulation in Theorem 3 can be turned into a formulation of polynomial size whenever we have the property that every point in $\operatorname{conv}\left(S^{(G, I)}\right)$ can be expressed as convex combination of points of $S^{(G, I)}$ in which the fractional parts of the coordinates of these points can take only "a small number" of possible values. More formally.

Remark 7 Suppose we are given $\mathcal{L} \subset\{0, \ldots, k-1\}$ with the property that

$$
\operatorname{conv}\left(S^{(G, I)}\right)=\operatorname{conv}\left(S^{(G, I)} \cap\left\{x \mid k\left(x_{i}-\left\lfloor x_{i}\right\rfloor\right) \in \mathcal{L}\right\}\right) .
$$

(By Lemma 1, the set $\{0, \ldots, k-1\}$ has the above property.)

The extended formulation presented in Section 2 can be turned into an extended formulation of size polynomial in $|\mathcal{L}|$ by setting $\mu_{i}^{t}=\mu_{i}^{t-1}$ whenever $k-t \notin \mathcal{L}$ and then eliminating variables and duplicate constraints.

In particular, whenever such a set $\mathcal{L}$ is known whose size is polynomial in the input data, this yields a polynomial size extended formulation. 
Conforti et al. [2] give bounds on the smallest size of a set $\mathcal{L}$ satisfying the conditions of Remark 7 . Given any such set $\mathcal{L}$, they also give an extended formulation for $\operatorname{conv}\left(S^{(G, I)}\right)$ that has $|\mathcal{L}||V(G)|$ additional variables. It can be seen that such an extended formulation is that given in Remark 7, with the additional constraints $\mu_{i}^{t}-\mu_{i}^{t-1} \geq 0, t=1, \ldots, k-1, \mu_{i}^{0}-\mu_{i}^{k-1} \geq-1$.

\section{Equivalence of bipartite vertex covers and dual network models}

Let $A$ be a totally unimodular matrix with two nonzero entries in each row. Let $N$ be the set of columns of $A$, and let $I \subseteq N$. Let $b$ be a rational vector and $\alpha, \beta \in \mathbb{Q}^{N}$. In this section we show how the problem of characterizing the convex hull of

$$
X^{2 T U}=\left\{x \in \mathbb{R}^{N} \mid A x \geq b, \alpha \leq x \leq \beta, x_{i} \text { integer, } i \in I\right\}
$$

can be reduced to the problem of characterizing the convex hull of a set of type $S^{(G, I)}$. We start by considering the special case in which $A$ has no negative $(-1)$ entries, and show how to deal with bounds on the integer variables and lower bounds on the continuous variables, before treating the general case.

If $A$ is a 0,1 matrix with two ones per row, then $A$ is totally unimodular if and only if it is the edge-node incidence matrix of some bipartite graph $G$, and therefore the set $S^{(G, I)}$ is a set of the type $X^{2 T U}$.

Bounds on variables First we show how to deal with bounds in the model $S^{(G, I)}$, namely we consider sets of the form $S^{(G, I)} \cap\{x \mid \alpha \leq x \leq \beta\}$ where $\alpha, \beta \in \mathbb{Q}^{U \cup V}$.

Lemma 8 Given $\alpha_{i}, \beta_{i} \in \mathbb{R} \cup\{ \pm \infty\}$ for every $i \in I$,

$\operatorname{conv}\left(S^{(G, I)} \cap\left\{x \mid \alpha_{i} \leq x_{i} \leq \beta_{i}, i \in I\right\}\right)=\operatorname{conv}\left(S^{(G, I)}\right) \cap\left\{x \mid\left\lceil\alpha_{i}\right\rceil \leq x_{i} \leq\left\lfloor\beta_{i}\right\rfloor, i \in I\right\}$.

Proof: The nontrivial inclusion to prove is that the set on the left contains the set on the right. Let $\bar{x} \in \operatorname{conv}\left(S^{(G, I)}\right) \cap\left\{x \mid\left\lceil\alpha_{i}\right\rceil \leq x_{i} \leq\left\lfloor\beta_{i}\right\rfloor, i \in I\right\}$. Then there exists $\bar{\mu}$ such that $(\bar{x}, \bar{\mu})$ is in the polyhedron $\mathcal{Q}_{I}$ defined in Theorem 3. In particular $\bar{\mu}$ satisfies the system defined by (2),(3),(4) and by the constraints $\left\lceil\alpha_{i}\right\rceil \leq \bar{\mu}_{i}^{t} \leq\left\lfloor\beta_{i}\right\rfloor, i \in I, t=0, \ldots, k-1$. The constraint matrix of the latter system is totally unimodular and the right-hand-side is integral, thus $\bar{\mu}$ is convex combination of integral points $\mu^{1}, \ldots, \mu^{h}$ satisfying (2),(3),(4), and $\left\lceil\alpha_{i}\right\rceil \leq \bar{\mu}_{i}^{t} \leq\left\lfloor\beta_{i}\right\rfloor, i \in I, t=0, \ldots, k-1$. Let $x^{1}, \ldots, x^{h}$ be the points defined by $\mu^{1}, \ldots, \mu^{h}$ in (1). Then $x^{1}, \ldots, x^{h}$ are elements of $S^{(G, I)} \cap\left\{x \mid \alpha_{i} \leq x_{i} \leq \beta_{i}, i \in I\right\}$ and $\bar{x}$ is a convex combination of $x^{1}, \ldots, x^{h}$. Thus $\bar{x} \in \operatorname{conv}\left(S^{(G, I)} \cap\left\{x \mid \alpha_{i} \leq x_{i} \leq \beta_{i}, i \in I\right\}\right)$. 
Given $Z \subseteq L$ and $\alpha_{i} \in \mathbb{Q}$ for every $i \in Z$, we observe next that the problem of characterizing the convex hull of $S^{(G, I)} \cap\left\{x \mid x_{i} \geq \alpha_{i}, i \in Z\right\}$ can be reduced to that of characterizing $\operatorname{conv}\left(S^{\left(G^{\prime}, I^{\prime}\right)}\right)$ for some suitable bipartite graph $G^{\prime}$ and a subset $I^{\prime}$ of its nodes. Graph $G^{\prime}$ is obtained as follows: let $Z^{\prime}$ be a copy of $Z$ disjoint from $U \cup V$, and let $i^{\prime}$ be the copy of $i$ in $Z^{\prime}$ for every $i$ in $Z$. Let $E^{Z}=E \cup\left\{i i^{\prime} \mid i \in Z\right\}, b_{e}^{\prime}=b_{e}$ for $e \in E, b_{i i^{\prime}}^{\prime}=\alpha_{i}, i \in Z^{\prime}$, and $I^{\prime}=I \cup Z^{\prime}$.

Lemma 9 Given $Z \subseteq L$ and $\alpha_{i} \in \mathbb{Q}$ for every $i \in Z$, then

$\operatorname{conv}\left(S^{(G, I)} \cap\left\{x \mid x_{i} \geq \alpha_{i}, i \in Z\right\}\right)=\operatorname{conv}\left(S^{\left(G^{\prime}, I^{\prime}\right)}\right) \cap\left\{x \in \mathbb{R}^{U \cup V \cup Z} \mid x_{i^{\prime}}=0, i \in Z\right\}$.

Proof. Note that $S^{(G, I)} \cap\left\{x \mid x_{i} \geq \alpha_{i}, i \in Z\right\}$ is the projection onto $\mathbb{R}^{U \cup V}$ of the set of vectors in $x \in \mathbb{R}^{U \cup V \cup Z^{\prime}}$ satisfying $x_{i}+x_{j} \geq b_{i j}^{\prime}$ for every $i j \in E^{Z}$, $x_{i} \in \mathbb{Z}, i \in I, x_{i^{\prime}}=0$ for every $i \in Z$. By Lemma 8, $\operatorname{conv}\left(S^{\left(G^{\prime}, I^{\prime}\right)} \cap\{x \in\right.$ $\left.\left.\mathbb{R}^{U \cup V \cup Z^{\prime}} \mid x_{i^{\prime}}=0, i \in Z\right\}\right)=\operatorname{conv}\left(S^{\left(G^{\prime}, I^{\prime}\right)}\right) \cap\left\{x \in \mathbb{R}^{U \cup V \cup Z^{\prime}} \mid x_{i^{\prime}}=0, i \in Z\right\}$. Therefore an inequality is valid for $S^{(G, I)} \cap\left\{x \mid x_{i} \geq \alpha_{i}, i \in Z\right\}$ if and only if it is obtained from some valid inequality for $S^{\left(G^{\prime}, I^{\prime}\right)}$ by replacing the variable $x_{i^{\prime}}$ with zero for every $i \in Z$.

Lemma 8 implies that adding a bound on an integer variable does not give rise to any new inequality. On the other hand, when $x_{i}$ is a continuous variable, the polyhedron $\operatorname{conv}\left(S^{(G, I)} \cap\left\{x \mid x_{i} \geq \alpha_{i}\right\}\right)$, that can be derived from $\operatorname{conv}\left(S^{\left(G^{\prime}, I^{\prime}\right)}\right)$ using Lemma 9, may have exponentially many more facets than $\operatorname{conv}\left(S^{(G, I)}\right)$.

Negative entries We now consider a general set of the form $X^{2 T U}$.

Let $N^{\prime}$ be a copy of $N$, and let $i^{\prime} \in N^{\prime}$ be the copy of $i \in N$. We define a system of inequalities in the variables $x_{i}, x_{i^{\prime}}, i \in N$. Specifically replace $-x_{i}$ by $x_{i^{\prime}}$ in every constraint of $A x \geq b$ in which $x_{i}$ appears with a -1 coefficient. Let $A^{+} x+A^{-} x^{\prime} \geq b, x_{i}+x_{i^{\prime}}=0, i \in N$, be the resulting system. Theorem 4 implies that the columns of $A$ can be partitioned into two sets $R$ and $B$ so that every row of $A$ has either a +1 and a -1 in the same side of the partition, or two $1 \mathrm{~s}$ or two $-1 \mathrm{~s}$ in distinct sides. Let $R^{+}$, $B^{+}$denote the set of columns of $A^{+}$corresponding to $R$ and $B$ respectively, and define $R^{-}, B^{-}$similarly for $A^{-}$. Then every row of

$$
\left(\begin{array}{cc}
A^{+} & A^{-} \\
I & I
\end{array}\right)
$$

has a 1 in $R^{+} \cup B^{-}$and a 1 in $R^{-} \cup B^{+}$. Since the constraint matrix of the above system has two ones per row, it is the edge-node incidence matrix of a bipartite graph $G_{A}$. We define $b_{i i^{\prime}}=0, i \in N$, while, if $e \in E\left(G_{A}\right)$ corresponds to the $h t h$ inequality of $A x \geq b$, we define $b_{e}^{\prime}=b_{h}$.

Proposition $10 \operatorname{conv}\left(\left\{x \in \mathbb{R}^{N} \mid A x \geq b, x_{i} \in \mathbb{Z}, i \in I\right\}\right)$ is the projection onto $R^{N}$ of $\operatorname{conv}\left(S^{\left(G_{A}, I\right)}\right) \cap\left\{x \in \mathbb{R}^{N \cup N^{\prime}} \mid x_{i}+x_{i^{\prime}}=0, i \in N\right\}$. 
Proof. Note that $\left\{x \in \mathbb{R}^{N} \mid A x \geq b, x_{i} \in \mathbb{Z}, i \in I\right\}$ is the projection onto $R^{N}$ of the points of $S^{\left(G_{A}, I\right)}$ satisfying $x_{i}+x_{i^{\prime}}=0, i \in N$. Since $x_{i}+x_{i^{\prime}} \geq 0$ define faces of $\operatorname{conv}\left(S^{\left(G_{A}, I\right)}\right)$, it follows that $\operatorname{conv}\left(S^{\left(G_{A}, I\right)} \cap\left\{x \in \mathbb{R}^{N \cup N^{\prime}} \mid x_{i}+x_{i^{\prime}}=\right.\right.$ $0, i \in N\})$ coincides with $\operatorname{conv}\left(S^{\left(G_{A}, I\right)}\right) \cap\left\{x \in \mathbb{R}^{N \cup N^{\prime}} \mid x_{i}+x_{i^{\prime}}=0, i \in N\right\}$.

Finally, we can express the constraints $x_{i} \leq \beta_{i}, i \in N$, by $x_{i^{\prime}} \geq-\beta_{i}$. By Lemmas 8 and 9, adding lower bounds on components of the points in $S^{\left(G_{A}, I\right)}$ can again be reduced to studying a set of the form $S^{\left(G^{\prime}, I^{\prime}\right)}$ for some suitable bipartite graph $G^{\prime}$ and subset of nodes $I^{\prime}$. Therefore studying the set $S^{(G, I)}$ is equivalent to studying the seemingly more general set $X^{2 T U}$.

We observe that the above discussion also allows us to give an extended formulation for $X^{2 T U}$ similar to that described in Theorem 3. Sometimes it will be more convenient to deal directly with such a formulation rather than appealing to Proposition 10, thus we give it explicitly.

Theorem 11 Let $G$ be a graph, I a subset of $V(G), b_{e}, e \in E(G)$ be rational numbers, and $E_{++}, E_{+-}, E_{--}$be a partition of $E(G)$. Let $X \subset \mathbb{R}^{E(G)}$ be the set of points satisfying

$$
\begin{array}{rl}
x_{i}+x_{j} & \geq b_{i j} \quad i j \in E_{++} \\
x_{i}-x_{j} & \geq b_{i j} \quad i j \in E_{+-} \\
-x_{i}-x_{j} & \geq b_{i j} \quad i j \in E_{--} \\
x_{i} \in \mathbb{Z} & i \in I .
\end{array}
$$

Assume that the constraint matrix of the above system is totally unimodular. Then the following is an extended formulation for $\operatorname{conv}(X)$.

$$
\begin{array}{rc}
x_{i}-\frac{1}{k}\left(\mu_{i}^{0}+\cdots+\mu_{i}^{k-1}\right)=0 & i \in U \cup V \\
\mu_{i}^{t}+\mu_{j}^{k-h_{i j}-1-t} \geq\left\lfloor b_{i j}\right\rfloor & t=0, \ldots, k-h_{i j}-1 ; \quad i j \in E_{++} \\
\mu_{i}^{t}+\mu_{j}^{2 k-h_{i j}-1-t} \geq\left\lceil b_{i j}\right\rceil & t=k-h_{i j}, \ldots, k-1 ; \quad i j \in E_{++} \\
\mu_{i}^{t}-\mu_{j}^{h_{i j}+t} \geq\left\lfloor b_{i j}\right\rfloor, & t=0, \ldots, k-h_{i j}-1 ; \quad i j \in E_{+-} \\
\mu_{i}^{t}-\mu_{j}^{h_{i j}-k+t} \geq\left\lceil b_{i j}\right\rceil, & t=k-h_{i j}, \ldots, k-1 ; \quad i j \in E_{+-} \\
-\mu_{i}^{t}-\mu_{j}^{h_{i j}-1-t} \geq\left\lceil b_{i j}\right\rceil & t=0, \ldots, h_{i j}-1 \quad i j \in E_{--} \\
-\mu_{i}^{t}-\mu_{j}^{k+h_{i j}-1-t} \geq\left\lfloor b_{i j}\right\rfloor & t=h_{i j}, \ldots, k-1 \quad i j \in E_{--} \\
\mu_{i}^{t-1}-\mu_{i}^{t}=0 & t=1, \ldots, k-1, i \in I .
\end{array}
$$

Proof. We discuss here constraint of the form $x_{i}-x_{j} \geq b_{i j}$. Applying the transformation described in Proposition 10, the latter becomes $x_{i}+x_{j^{\prime}} \geq b_{i j}$, $x_{j}+x_{j^{\prime}}=0$, therefore the extended formulation is 


$$
\begin{gathered}
x_{i}-\frac{1}{k}\left(\mu_{i}^{0}+\cdots+\mu_{i}^{k-1}\right)=0 \\
x_{j}-\frac{1}{k}\left(\mu_{j}^{0}+\cdots+\mu_{j}^{k-1}\right)=0 \\
x_{j^{\prime}}-\frac{1}{k}\left(\mu_{j^{\prime}}^{0}+\cdots+\mu_{j^{\prime}}^{k-1}\right)=0 \\
\mu_{i}^{t}+\mu_{j^{\prime}}^{q_{i j t}} \geq\left\lfloor b_{i j}\right\rfloor, \quad t=0, \ldots, k-h_{i j}-1 ; \\
\mu_{i}^{t}+\mu_{j^{\prime}}^{q_{i j t}} \geq\left\lceil b_{i j}\right\rceil, \quad t=k-h_{i j}, \ldots, k-1 . \\
\mu_{j^{\prime}}^{t}+\mu_{j}^{k-t-1}
\end{gathered}
$$

Substituting $-\mu_{j}^{k-t-1}$ for $\mu_{j^{\prime}}^{t}$, we can express the previous constraints only in terms of $x_{i}, x_{j}, \mu_{i}, \mu_{j}$, namely

$$
\begin{gathered}
x_{i}-\frac{1}{k}\left(\mu_{i}^{0}+\cdots+\mu_{i}^{k-1}\right)=0 \\
x_{j}-\frac{1}{k}\left(\mu_{j}^{0}+\cdots+\mu_{j}^{k-1}\right)=0 \\
\mu_{i}^{t}-\mu_{j}^{h_{i j}+t} \\
\mu_{i}^{t}-\mu_{j}^{h_{i j}-k+t}
\end{gathered}
$$

Similarly one can treat constraints of the form $-x_{i}-x_{j} \geq b_{i j}$.

Example. To illustrate Theorem 11, consider the set

$$
\left\{x \in \mathbb{R}^{4},-x_{1}+x_{2} \geq 3 / 4,-x_{2}-x_{3} \geq 1 / 4, x_{3}-x_{4} \geq 2 / 4, x_{4} \in \mathbb{Z}\right\} .
$$

The extended formulation is depicted in Figure 3. Each edge corresponds to an inequality, a minus sign on an edge indicates that the coefficient of the corresponding $\mu_{i}^{t}$ variable in the inequality is -1 , and the number indicates the right-hand side.

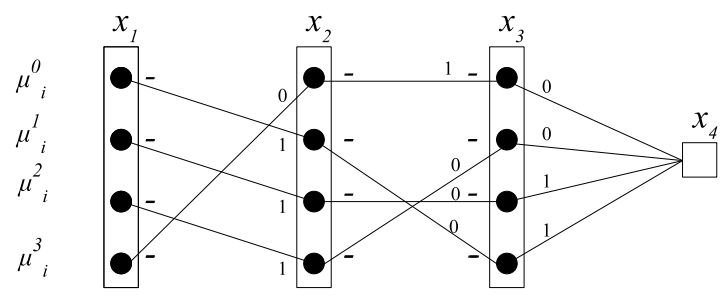

Figure 3: Extended formulation in the example. 


\section{On the projection of the extended formulation}

By Theorem 3 and Remark $5, \operatorname{conv}\left(S^{(G, I)}\right)$ is the projection onto the space of the $x$ variables of the polyhedron described by the following inequalities.

$\left(c_{i}\right) \quad k x_{i}-\mu_{i}^{0}-\cdots-\mu_{i}^{k-1}=0, \quad i \in L$

$\left(u_{i j}^{t}\right) \quad \mu_{i}^{t}+\mu_{j}^{q_{i j t}} \geq\left\lfloor b_{i j}\right\rfloor, \quad t=0, \ldots, k-h_{i j}-1, i j \in E, i, j \in L ;$

$\left(u_{i j}^{t}\right) \mu_{i}^{t}+\mu_{j}^{q_{i j t}} \geq\left\lceil b_{i j}\right\rceil, \quad t=k-h_{i j}, \ldots, k-1, i j \in E, i, j \in L ;$

$\left(u_{i j}^{t}\right) \quad \mu_{i}^{t}+x_{j} \geq\left\lfloor b_{i j}\right\rfloor, \quad t=0, \ldots, k-h_{i j}-1, i j \in E, i \in L, j \in I ;$

$\left(u_{i j}^{t}\right) \quad \mu_{i}^{t}+x_{j} \geq\left\lceil b_{i j}\right\rceil, \quad t=k-h_{i j}, \ldots, k-1, i j \in E, i \in L, j \in I$;

$\left(w_{i j}\right) \quad x_{i}+x_{j} \geq\left\lceil b_{i j}\right\rceil, \quad i j \in E, i, j \in I$.

For each $i j \in E$ and $t=0, \ldots, k-1$, let $\beta_{i j}^{t}$ be the right-hand-side of the inequalities relative to edges $i j$ with $i \in L$. Notice that to each inequality we have associated a multiplier, written on the left between parentheses. Because of the symmetry between indices $i$ and $j$ in the inequality $\mu_{i}^{t}+\mu_{j}^{q_{i j t}} \geq$ $\beta_{i j}$, we identify the multipliers $u_{i j}^{t}$ and $u_{j i}^{q_{i j t}}$ for every $i j \in E, i, j \in L$, and $t=0, \ldots, k-1$.

Any valid inequality for $S^{(G, I)}$ is determined by a vector of multipliers $(c, u, w)$, and has the form $\pi^{(c, u, w)} x \geq \delta^{(c, u, w)}$ where

$$
\begin{aligned}
& \pi^{(c, u, w)} x=\sum_{i \in L} k c_{i} x_{i}+\sum_{j \in I}\left(\sum_{\substack{i \in L: \\
i j \in E}} \sum_{t=0}^{k-1} u_{i j}^{t}\right) x_{j}+\sum_{i \in I}\left(\sum_{\substack{j \in I: \\
i j \in E}} w_{i j}\right) x_{i}, \\
& \delta^{(c, u, w)}=\sum_{t=0}^{k-1} \sum_{\substack{i j \in E: \\
i \in L}} u_{i j}^{t} \beta_{i j}^{t}+\sum_{\substack{i j \in E: \\
i, j \in I}} w_{i j}\left\lceil b_{i j}\right\rceil,
\end{aligned}
$$

when $(c, u, w)$ satisfy

$$
\begin{aligned}
\sum_{j: i j \in E} u_{i j}^{t} & =c_{i}, \quad i \in L, t=0, \ldots, k-1 ; \\
u_{i j}^{t} & \geq 0, \quad i j \in E, t=0, \ldots, k-1 ; \\
w_{i j} & \geq 0, \quad i j \in E, i, j \in I .
\end{aligned}
$$

(See Theorem 4.10 in [9]). 
Example. Consider the following problem

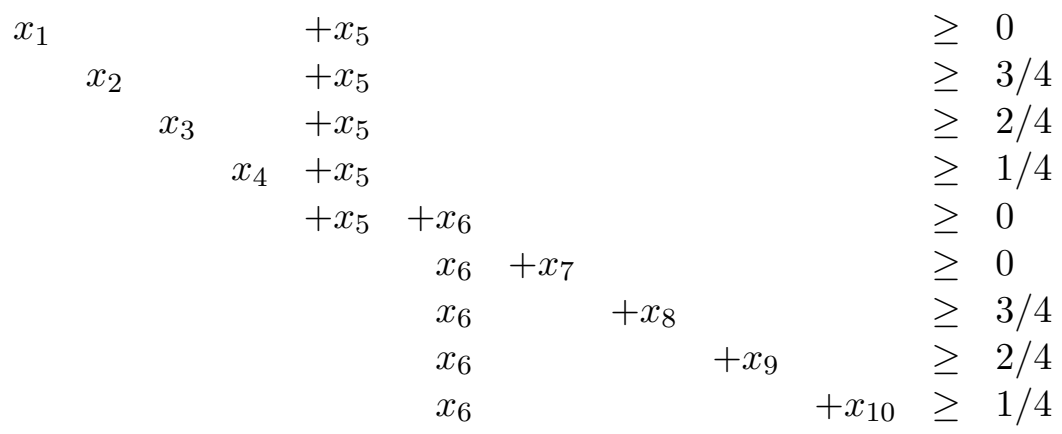

$$
\begin{aligned}
& x_{1}, x_{2}, x_{3}, x_{4}, x_{7}, x_{8}, x_{9}, x_{10} \in \mathbb{Z}, x_{5}, x_{6} \in \mathbb{R}
\end{aligned}
$$

represented in Figure 4.

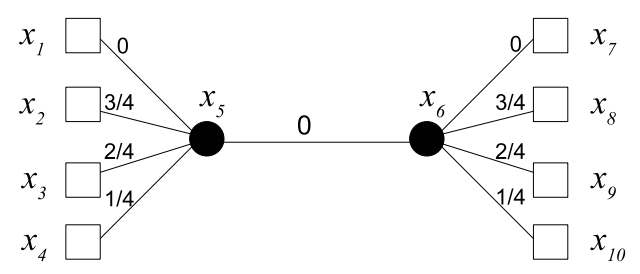

Figure 4: Original problem. Square nodes correspond to integer variables.

The extended formulation is given by

$$
\begin{array}{rrrl}
4 x_{i} & -\sum_{j=0}^{3} \mu_{i}^{t} & =0 & i=5,6 \\
x_{i} & \mu_{5}^{t} & \geq \beta_{5 i}^{t} \quad i=1,2,3,4 ; t=0,1,2,3 ; \\
\mu_{5}^{3-t} & \geq 0 \quad t=0,1,2,3 ; \\
\mu_{6}^{t} & \mu_{6}^{t}+x_{i} & \geq \beta_{6 i}^{t} \quad i=7,8,9,10 ; t=0,1,2,3 ;
\end{array}
$$

and is depicted in Figure 5.

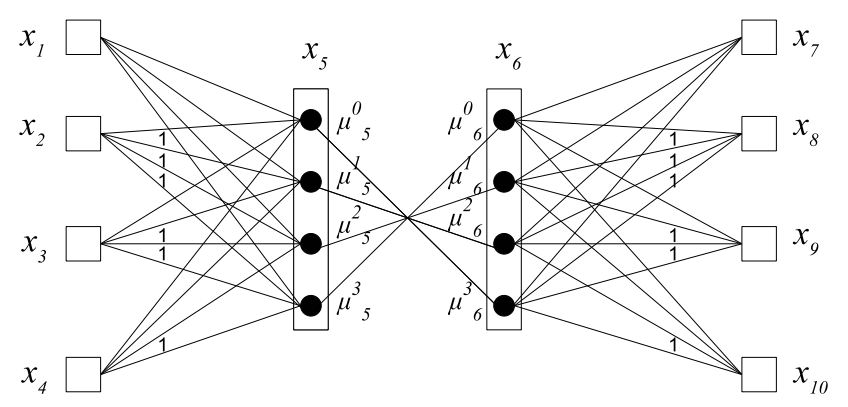

Figure 5: Extended problem. Non-zero requirements on the edges are shown. 
Consider the vector of multipliers $(c, u)$ where $c_{5}=1, u_{52}^{t}=1$ for $t=$ $0,1,2,3$ and all other entries are equal to 0 that satisfies (6). Then the corresponding inequality (5) is $4 x_{2}+4 x_{5} \geq 3$, which is one of the original inequalities.

As another example, let $(c, u)$ be given by $c_{5}=c_{6}=1, u_{56}^{0}=u_{56}^{2}=1$, $u_{52}^{1}=u_{54}^{3}=u_{67}^{0}=u_{69}^{2}=1$, and all other entries equal to 0 . Then the corresponding inequality (5) is $x_{2}+x_{4}+4 x_{5}+4 x_{6}+x_{7}+x_{9} \geq 3$.

Remark 12 If $G^{\prime}$ is a subgraph of $G$ and $I^{\prime}=V\left(G^{\prime}\right) \cap I$, a valid inequality for $\operatorname{conv}\left(S^{\left(G^{\prime}, I^{\prime}\right)}\right)$ is also valid for $\operatorname{conv}\left(S^{(G, I)}\right)$.

We are interested in characterizing the vectors $(c, u, w)$ satisfying $(6)$ for which $\pi^{(c, u, w)} x \geq \delta^{(c, u, w)}$ is facet-defining for $\operatorname{conv}\left(S^{(G, I)}\right)$, but is not one of the original inequalities $x_{i}+x_{j} \geq b_{i j}, i j \in E$. Let $\tilde{E}$ be the set of edges $i j \in E$ such that $u_{i j}^{t}>0$ for some $t \in\{0, \ldots, k-1\}$ or $w_{i j}>0$. Let $\tilde{G}$ be the subgraph of $G$ whose edges are the elements of $\tilde{E}$ and whose nodes are the endnodes of edges in $\tilde{E}$. We call $\tilde{G}$ the skeleton of $(c, u, w)$.

Remark 13 If the inequality $\pi^{(c, u, w)} x \geq \delta^{(c, u, w)}$ is facet-defining for $\operatorname{conv}\left(S^{(G, I)}\right)$, then it is facet-defining for $\operatorname{conv}\left(S^{(\tilde{G}, \tilde{I})}\right)$, where $\tilde{I}=V(\tilde{G}) \cap I$.

Proof. Note that $\pi^{(c, u, w)} x \geq \delta^{(c, u, w)}$ is valid for $\operatorname{conv}\left(S^{(\tilde{G}, \tilde{I})}\right)$, since $u_{i j}^{t}, w_{i j}$ are zero on the edges $i j$ of $E(G) \backslash E(\tilde{G})$ and $c_{i}=0$ for every $i \in V(G) \backslash V(\tilde{G})$. If $\pi^{(c, u, w)} x \geq \delta^{(c, u, w)}$ is facet-defining for $\operatorname{conv}\left(S^{(G, I)}\right)$, it is satisfied at equality by $|V(G)|$ affinely independent points $x^{1}, \ldots, x^{|V(G)|}$ in $S^{(G, I)}$. Let $\bar{x}^{1}, \ldots, \bar{x}^{|V(G)|}$ be the restriction of $x^{1}, \ldots, x^{|V(G)|}$ to the components relative to nodes in $V(\tilde{G})$. Then, by standard linear algebra, $\bar{x}^{1}, \ldots, \bar{x}^{|V(G)|}$ contain $|V(\tilde{G})|$ affinely independent vectors.

In the light of Remarks 12 and 13, we may assume that $G$ coincides with the skeleton $\tilde{G}$ of $(c, u, w)$.

Notice that the equations in (6) are defined only for the nodes $i \in L$. This implies the following:

Lemma 14 Let $E^{\prime}, E^{\prime \prime}$ be a partition of the edges of $E$ such that, for every node $i \in L$, the edges incident to $i$ are either all in $E^{\prime}$ or all in $E^{\prime \prime}$. Let $G^{\prime}=\left(U \cup V, E^{\prime}\right)$ and $G^{\prime \prime}=\left(U \cup V, E^{\prime \prime}\right)$. Then $\operatorname{conv}\left(S^{(G, I)}\right)=\operatorname{conv}\left(S^{\left(G^{\prime}, I\right)}\right) \cap$ $\operatorname{conv}\left(S^{\left(G^{\prime \prime}, I\right)}\right)$.

Proof: Clearly $\operatorname{conv}\left(S^{(G, I)}\right) \subseteq \operatorname{conv}\left(S^{\left(G^{\prime}, I\right)}\right) \cap \operatorname{conv}\left(S^{\left(G^{\prime \prime}, I\right)}\right)$. Next we show the reverse containment.

Let $\pi^{(c, u, w)} x \geq \delta^{(c, u, w)}$ be defined by multipliers $(c, u, w)$ satisfying (6). Define vectors $\left(c^{\prime}, u^{\prime}, w^{\prime}\right),\left(c^{\prime \prime}, u^{\prime \prime}, w^{\prime \prime}\right)$ as follows. $c_{i}^{\prime}=c_{i}, c_{i}^{\prime \prime}=0$ if $i \in L$ is 
adjacent only to edges in $E^{\prime}, c_{i}^{\prime}=0, c_{i}^{\prime \prime}=c_{i}$ if $i \in L$ is adjacent only to edges in $E^{\prime \prime} ; u_{i j}^{\prime t}=u_{i j}^{t}, u_{i j}^{\prime \prime t}=0, w_{i j}^{\prime}=w_{i j}, w_{i j}^{\prime \prime}=0$, if $i j \in E^{\prime}$; $u_{i j}^{\prime t}=0, u_{i j}^{\prime \prime}=u_{i j}^{t}, w_{i j}^{\prime}=0, w_{i j}^{\prime \prime}=w_{i j}$, if $i j \in E^{\prime \prime}$. Notice that $(c, u, w)=$ $\left(c^{\prime}, u^{\prime}, w^{\prime}\right)+\left(c^{\prime \prime}, u^{\prime \prime}, w^{\prime \prime}\right)$, and, by our assumptions on $E^{\prime}$ and $E^{\prime \prime},\left(c^{\prime}, u^{\prime}, w^{\prime}\right)$ and $\left(c^{\prime \prime}, u^{\prime \prime}, w^{\prime \prime}\right)$ satisfy (6). Furthermore, since $\left(c^{\prime}, u^{\prime}, w^{\prime}\right)$ is zero on all entries relative to edges in $E^{\prime \prime}$ and $\left(c^{\prime \prime}, u^{\prime \prime}, w^{\prime \prime}\right)$ is zero on all entries relative to edges in $E^{\prime}, \pi^{\left(c^{\prime}, u^{\prime}, w^{\prime}\right)} x \geq \delta^{\left(c^{\prime}, u^{\prime}, w^{\prime}\right)}$ and $\pi^{\left(c^{\prime \prime}, u^{\prime \prime}, w^{\prime \prime}\right)} x \geq \delta^{\left(c^{\prime \prime}, u^{\prime \prime}, w^{\prime \prime}\right)}$ are valid for $\operatorname{conv}\left(S^{\left(G^{\prime}, I\right)}\right)$ and $\operatorname{conv}\left(S^{\left(G^{\prime \prime}, I\right)}\right)$, respectively. Hence $\pi^{(c, u, w)} x \geq \delta^{(c, u, w)}$ can be written as nonnegative combination of inequalities valid for $\operatorname{conv}\left(S^{\left(G^{\prime}, I\right)}\right)$ or $\operatorname{conv}\left(S^{\left(G^{\prime \prime}, I\right)}\right)$, thus the statement follows.

Because of Lemma 14, we assume the following from now on:

A1) $G$ has no edge $i j$ with $i, j \in I$;

A2) There is no node-cutset $S$ of $G$ with $S \subseteq I$.

Indeed, if $i j$ is an edge with $i, j \in I$, then $E^{\prime}=\{i j\}, E^{\prime \prime}=E \backslash\{i j\}$ satisfy the hypothesis of Lemma 14, while if $S \subseteq I$ is a cutset of $G$, then the edges of $G$ can be partitioned into two subsets $E^{\prime}$ and $E^{\prime \prime}$ satisfying the hypothesis of Lemma 14.

Under assumption A1), the vector $w$ disappears in (6) and the inequality defined by (5) becomes

$$
\sum_{i \in L} k c_{i} x_{i}+\sum_{j \in I}\left(\sum_{\substack{i \in L: \\ i j \in E}}^{k-1} \sum_{t=0}^{t} u_{i j}^{t}\right) x_{j} \geq \sum_{t=0}^{k-1} \sum_{\substack{i j \in E \\ i \in L}} u_{i j}^{t} \beta_{i j}^{t} .
$$

We will denote such an inequality by $\pi^{(c, u)} x \geq \delta^{(c, u)}$. Without loss of generality, we will assume that:

A3) $(c, u)$ is integral, and its entries are co-prime.

The following remarks explains the use of assumption A3).

Remark 15 Let $(c, u)$ be an integral vector with coprime entries satisfying (6). If there exist $\left(c^{\prime}, u^{\prime}\right)$ and $\left(c^{\prime \prime}, u^{\prime \prime}\right)$ satisfying (6) such that $(c, u)=$ $\left(c^{\prime}, u^{\prime}\right)+\left(c^{\prime \prime}, u^{\prime \prime}\right)$, then $\pi^{(c, u)} x \geq \delta^{(c, u)}$ is not facet-defining.

Since we assume that $G$ coincides with the skeleton of $(c, u)$, the following holds.

A4) For every $i j \in E$, there is a $t \in\{0, \ldots, k-1\}$ such that $u_{i j}^{t}>0$. 
Assume that, for some edge $i j \in E, u_{i j}^{t}>0$ for $t=0, \ldots, k-1$. Let $u_{i j}^{\prime t}=u_{i j}^{t}-1$, for $t=0, \ldots, k-1$, and $u_{h k}^{\prime t}=u_{h k}^{t}$, for $h k \in E \backslash\{i j\}$, $t=0, \ldots, k-1$. Let $c_{i}^{\prime}=c_{i}-1$ if $i \in L, c_{j}^{\prime}=c_{j}-1$ if $j \in L$, and $c_{h}^{\prime}=c_{h}$ for $h \in L \backslash\{i, j\}$. We have that $\left(c^{\prime}, u^{\prime}\right)$ satisfy (6). Furthermore, $\pi^{(c, u)} x \geq \delta^{(c, u)}$ is the sum of the inequality $\pi^{\left(c^{\prime}, u^{\prime}\right)} x \geq \delta^{\left(c^{\prime}, u^{\prime}\right)}$ and $k x_{i}+k x_{j} \geq k b_{i j}$. Since we are assuming that $\pi^{(c, u)} x \geq \delta^{(c, u)}$ is facet-defining, then by A3) it must be precisely the inequality $k x_{i}+k x_{j} \geq k b_{i j}$. Hence we can assume the following:

A5) For every $i j \in E$, there is a $t \in\{0, \ldots, k-1\}$ such that $u_{i j}^{t}=0$.

\subsection{Facets defined by trees}

In this section we focus our attention on the multipliers $(c, u)$ whose skeleton is a tree, namely the case where $G$ is a tree. We will assume that $(c, u)$ satisfies A1)-A5) and $\pi^{(c, u)} x \geq \delta^{(c, u)}$ is facet-defining for $\operatorname{conv}\left(S^{(G, I)}\right)$.

Remark 16 The set I coincides with the set of leaves of $G$.

Proof: By assumption A2), every node in $I$ must be a leaf of $G$, because every node of $G$ that is not a leaf is a cut-node of $G$. On the other hand, suppose there exists a node $i \in L$ that is a leaf of $G$. Let $j$ be the unique neighbor of $i$ in $G$. Since $(c, u)$ satisfies $(6)$, then $c_{i}=u_{i j}^{t}$ for $t=0, \ldots, k-1$. By assumption A4), we must have $u_{i j}^{t}>0$ for $t=0, \ldots, k-1$. This contradicts assumption A5).

Given $i \in I$, we will denote by $n(i)$ the unique neighbor of $i$ in $G$. By assumption A1), $n(i) \in L$ for every $i \in I$.

Since $G$ is a tree, we can reduce the requirements $b_{e}, e \in E$, to a simpler form by a change of variables. Specifically, choose a root node $r \in L$. For every node $i$ distinct from $r$, denote by $P_{i}$ the unique path from $r$ to $i$ in $G$, and by $p(i)$ the unique neighbor of $i$ in $P_{i}$. For every node $i$, define the number $\gamma_{i}$ by induction on the distance of $i$ from $r$, starting with $\gamma_{r}=0$ and defining $\gamma_{i}=b_{p(i) i}-\gamma_{p(i)}$.

For every $i \in L$, define the new variable $x_{i}^{\prime}=x_{i}-\gamma_{i}$, while for every $i \in I$ we define the new variable $x_{i}^{\prime}=x_{i}-\left\lfloor\gamma_{i}\right\rfloor$. Notice that, for every $i \in I$, $x_{i}$ has integer value if and only if $x_{i}^{\prime}$ has integer value. For every $i j \in E$ such that $i, j \in L$, the inequality $x_{i}+x_{j} \geq b_{i j}$ becomes $x_{i}^{\prime}+x_{j}^{\prime} \geq 0$.

For every $i \in I$, the inequality $x_{i}+x_{n(i)} \geq b_{i j}$ becomes $x_{i}^{\prime}+x_{n(i)}^{\prime} \geq \gamma_{i}-\left\lfloor\gamma_{i}\right\rfloor$.

Remark 17 We can assume that $b_{i j}=0$ for every $i j \in E$ with $i, j \in L$, and that $0 \leq b_{i j}<1$ otherwise.

In the light of the previous remark, we may assume that $b_{n(i) i}=h_{i} / k$ for every $i \in I$, where $k$ is an integer and $h_{i}$ is an integer between 0 and 
$k-1$. Under these assumptions, the extended formulation described at the beginning of this section becomes:

$$
\begin{array}{ll}
\left(c_{i}\right) & k x_{i}-\mu_{i}^{0}-\cdots-\mu_{i}^{k-1}=0, \quad i \in L \\
\left(u_{i j}^{t}\right) & \mu_{i}^{t}+\mu_{j}^{k-t-1} \geq 0, \quad t=0, \ldots, k-1, i j \in E, i, j \in L ; \\
\left(u_{n(i) i}^{t}\right) & \mu_{n(i)}^{t}+x_{i} \geq 0, \quad t=0, \ldots, k-h_{i}-1, i \in I \\
\left(u_{n(i) i}^{t}\right) & \mu_{n(i)}^{t}+x_{i} \geq 1, \quad t=k-h_{i}, \ldots, k-1, i \in I .
\end{array}
$$

For every $i \in I$, we denote by $\beta_{i}^{t}$ the right-hand-side of the inequality with multiplier $u_{n(i) i}^{t}$, namely $\beta_{i}^{t}=0$ for $t=0, \ldots, k-h_{i}-1$ and $\beta_{i}^{t}=1$ for $t=k-h_{i}, \ldots, k-1$.

The inequality $\pi^{(c, u)} x \geq \delta^{(c, u)}$ defined by (7) becomes

$$
\sum_{i \in L} k c_{i} x_{i}+\sum_{i \in I}\left(\sum_{t=0}^{k-1} u_{n(i) i}^{t}\right) x_{i} \geq \sum_{i \in I} \sum_{t=k-h_{i}}^{k-1} u_{n(i) i}^{t} .
$$

Notice that the right-hand-side of (8) can also be written $\sum_{i \in I} \sum_{t=0}^{k-1} \beta_{i}^{t} u_{n(i) i}^{t}$.

Given a node $r$ of $G$, an $r$-branch of $G$ is any subgraph induced by $\{r\} \cup C$ where $C$ is a connected component of $G \backslash r$. Two $r$-branches $T$ and $T^{\prime}$ of $G$ are said to be specular if there exists a graph isomorphism $\phi: V(T) \rightarrow V\left(T^{\prime}\right)$ such that $\phi(r)=r$ and $b_{n(i) i}=b_{\phi(n(i)) \phi(i)}$ for every node $i \in I \cap V(T)$.

Lemma 18 For any noder, $G$ does not have two distinct specular $r$-branches.

Proof: By contradiction, let $T, T^{\prime}$ be specular $r$-branches, where $r$ is a node of $G$. Notice that $r \in L$. Define vectors $u^{\prime}, u^{\prime \prime}$ as follows.

$$
\begin{aligned}
& u_{i j}^{\prime t} \quad=u_{i j}^{t}+u_{\phi(i) \phi(j)}^{t}, \quad t=0, \ldots, k-1, i j \in E(T), i \in L ; \\
& u_{\phi(i) \phi(j)}^{\prime t}=0, \quad t=0, \ldots, k-1, i j \in E(T), i \in L \text {; } \\
& {u^{\prime}}_{i j}^{t}=u_{i j}^{t}, \quad i j \notin E(T) \cup E\left(T^{\prime}\right) \text {. } \\
& \begin{array}{lll}
u_{i j}^{\prime \prime t} & =0, & t=0, \ldots, k-1, i j \in E(T), i \in L ; \\
u_{\phi(i) \phi(j)}^{\prime \prime t} & =u_{i j}^{t}+u_{\phi(i) \phi(j)}^{t}, & t=0, \ldots, k-1, i j \in E(T), i \in L
\end{array} \\
& u_{i j}^{\prime \prime}{ }_{i j} \quad=u_{i j}^{t}, \quad i j \notin E(T) \cup E\left(T^{\prime}\right) \text {. }
\end{aligned}
$$

Notice that $\left(c, u^{\prime}\right),\left(c, u^{\prime \prime}\right)$ satisfy (6). Also notice that $\pi_{i}^{\left(c, u^{\prime}\right)}=\pi_{i}^{(c, u)}+$ $\pi_{\phi(i)}^{(c, u)}, \pi_{\phi(i)}^{\left(c, u^{\prime}\right)}=0, \pi_{\phi(i)}^{\left(c, u^{\prime \prime}\right)}=\pi_{i}^{(c, u)}+\pi_{\phi(i)}^{(c, u)}, \pi_{i}^{\left(c, u^{\prime \prime}\right)}=0$ for every $i \in V(T) \backslash\{r\}$, while $\pi_{i}^{\left(c, u^{\prime}\right)}=\pi_{i}^{\left(c, u^{\prime \prime}\right)}=\pi_{i}^{(c, u)}$ for every other node of $G$. Thus, for any $i \in$ $V(T) \backslash\{r\}$, the numbers $\lambda^{\prime}=\pi_{i}^{(c, u)} /\left(\pi_{i}^{(c, u)}+\pi_{\phi(i)}^{(c, u)}\right)$ and $\lambda^{\prime \prime}=\pi_{\phi(i)}^{(c, u)} /\left(\pi_{i}^{(c, u)}+\right.$ $\left.\pi_{\phi(i)}^{(c, u)}\right)$ are independent of the choice of $i$. Furthermore $\pi^{(c, u)}=\lambda^{\prime} \pi^{\left(c, u^{\prime}\right)}+$ $\lambda^{\prime \prime} \pi^{\left(c, u^{\prime \prime}\right)}, \delta^{(c, u)}=\lambda^{\prime} \delta^{\left(c, u^{\prime}\right)}+\lambda^{\prime \prime} \delta^{\left(c, u^{\prime \prime}\right)}$. Since $\pi^{\left(c, u^{\prime}\right)} \neq \pi^{(c, u)}, \pi^{(c, u)} x \geq \delta^{(c, u)}$ cannot be facet-defining. 
Consider the inequality $x_{n(i)}+x_{i} \geq h_{i} / k$ for some $i \in I$. In the extended formulation, this inequality corresponds to the $k$ inequalities $\mu_{n(i)}^{t}+x_{i} \geq 0$, $t=0, \ldots, k-1-h_{i}, \mu_{n(i)}^{t}+x_{i} \geq 1, t=k-h_{i}, \ldots, k-1$. The next lemma shows that the multipliers $u_{n(i) i}^{t}$ associated with these $k$ inequalities are cyclically non-increasing starting from $u_{n(i) i}^{k-h_{i}}$, and this is a key property in characterizing facet-defining inequalities.

Lemma 19 For every node $i \in I$,

$$
u_{n(i) i}^{k-h_{i}} \geq u_{n(i) i}^{k-h_{i}+1} \geq \ldots \geq u_{n(i) i}^{k-1} \geq u_{n(i) i}^{0} \geq \ldots \geq u_{n(i) i}^{k-h_{i}-1} .
$$

Proof: Given $i_{0} \in I$, let $i_{1}=n\left(i_{0}\right)$. Assume $\tau, \tau^{\prime} \in\{0, \ldots, k-1\}$ are indices such that $u_{i_{1} i_{0}}^{\tau}>u_{i_{1} i_{0}}^{\tau^{\prime}}$.

Claim: There exists a path $i_{0}, i_{1}, \ldots, i_{s}, i_{s+1}$ in $G$, where $i_{1}, \ldots, i_{s} \in L$, $i_{s+1} \in I$, such that:

- For $r=1, \ldots, s, u_{i_{r} i_{r-1}}^{\tau}>u_{i_{r} i_{r-1}}^{\tau^{\prime}}$ if $r$ is odd, $u_{i_{r} i_{r-1}}^{k-1-\tau}<u_{i_{r} i_{r-1}}^{k-1-\tau^{\prime}}$ if $r$ is even;

- $u_{i_{s} i_{s+1}}^{\tau}<u_{i_{s} i_{s+1}}^{\tau^{\prime}}$ if $s$ is odd, $u_{i_{s} i_{s+1}}^{k-1-\tau}>u_{i_{s} i_{s+1}}^{k-1-\tau^{\prime}}$ if $s$ is even.

Let $i_{0}, i_{1}, \ldots, i_{s}$ be a path in $G$, that is maximal with the properties that $i_{1}, \ldots, i_{s} \in L$, and that $u_{i_{r} i_{r-1}}^{\tau}>u_{i_{r} i_{r-1}}^{\tau^{\prime}}$ if $r$ is odd, $u_{i_{r} i_{r-1}}^{k-1-\tau}<u_{i_{r} i_{r-1}}^{k-1-\tau^{\prime}}$ if $r$ is even, $r=1, \ldots, s$. Clearly $i_{0}, i_{1}$ is a path with such properties. Since $(c, u)$ satisfy

$$
\sum_{j: i_{s} j \in E} u_{i_{s} j}^{t}=c_{i_{s}}
$$

for every $t \in\{0, \ldots, k-1\}$, there exists a node $i_{s+1}$, adjacent to $i_{s}$ in $G$, such that $u_{i_{s} i_{s+1}}^{\tau}<u_{i_{s} i_{s+1}}^{\tau^{\prime}}$ if $s$ is odd, and $u_{i_{s} i_{s+1}}^{k-1-\tau}>u_{i_{s} i_{s+1}}^{k-1-\tau^{\prime}}$ if $s$ is even. If $i_{s+1} \in I$, then the statement is proven. Suppose then that $i_{s+1} \in L$. Thus (since $u_{i j}^{t}=u_{i j}^{k-t-1}$ for every $i j \in E$ ) $u_{i_{s+1}^{\tau} i_{s}}^{{ }^{\prime}}>u_{i_{s+1} i_{s}}^{\tau^{\prime}}$ if $s+1$ is odd, and $u_{i_{s+1} i_{s}}^{k-1-\tau}<u_{i_{s+1} i_{s}}^{k-1-\tau^{\prime}}$ if $s+1$ is even. Therefore we can increase the path $i_{0}, \ldots, i_{s}$ by adding the element $i_{s+1}$, contradicting the maximality assumption. This concludes the proof of the claim.

Suppose now that there exists a node $i_{0} \in I$ that contradicts the statement of the lemma. Let $i_{1}=n\left(i_{0}\right)$. Then there exist two indices $\tau, \tau^{\prime}$ such that $u_{i_{1} i_{0}}^{\tau}>u_{i_{1} i_{0}}^{\tau^{\prime}}$ and such that $\tau, \tau^{\prime}$ satisfy one of the following:

a) $\tau \in\left\{0, \ldots, k-h_{i_{0}}-1\right\}, \tau^{\prime} \in\left\{k-h_{i_{0}}, \ldots, k-1\right\}$.

b) $\tau>\tau^{\prime}$ and either $\tau, \tau^{\prime} \in\left\{0, \ldots, k-h_{i_{0}}-1\right\}$ or $\tau, \tau^{\prime} \in\left\{k-h_{i_{0}}, \ldots, k-1\right\}$. 
Then there exists a path $P=i_{0}, \ldots, i_{s+1}$ as in the statement of the previous Claim. We define two vectors $v$ and $w$ as follows:

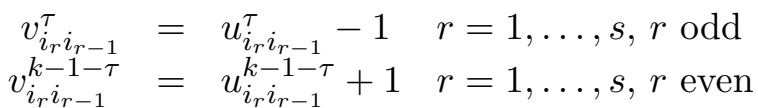

$$
\begin{aligned}
& v_{i_{s} i_{s+1}}^{\tau}=u_{i_{s} i_{s+1}}^{\tau}+1 \quad \text { if } s \text { odd } \\
& v_{i_{s} i_{s+1}}^{k-1-\tau}=u_{i_{s} i_{s+1}}^{k-1-\tau}-1 \text { if } s \text { even } \\
& v_{i j}^{t}=u_{i j}^{t} \quad \text { otherwise } \\
& w_{i_{r} i_{r-1}}^{\tau^{\prime}}=u_{i_{r} i_{r-1}}^{\tau^{\prime}}+1 \quad r=1, \ldots, s, r \text { odd } \\
& w_{i_{r} i_{r-1}}^{k-1-\tau^{\prime}}=u_{i_{r} i_{r-1}}^{k-1-\tau^{\prime}}-1 \quad r=1, \ldots, s, r \text { even } \\
& w_{i_{s} i_{s+1}}^{\tau^{\prime}}=u_{i_{s} i_{s+1}}^{\tau^{\prime}}-1 \quad \text { if } s \text { odd } \\
& w_{i_{s} i_{s+1}}^{k-1-\tau^{\prime}}=u_{i_{s} i_{s+1}}^{k-1-\tau^{\prime}}+1 \text { if } s \text { even } \\
& w_{i j}^{t}=u_{i j}^{t} \quad \text { otherwise }
\end{aligned}
$$

One can verify that $(c, v)$ and $(c, w)$ both satisfy conditions (6). Furthermore, $\pi_{i}^{(c, u)}=\pi_{i}^{(c, v)}=\pi_{i}^{(c, w)}$ for every node $i$ distinct from $i_{0}$ and $i_{s+1}$, while $\pi_{i_{0}}^{(c, v)}=\pi_{i_{0}}^{(c, u)}-1$ and $\pi_{i_{0}}^{(c, w)}=\pi_{i_{0}}^{(c, u)}+1$.

If $s$ is odd, then $\pi_{i_{s+1}}^{(c, v)}=\pi_{i_{s+1}}^{(c, u)}+1, \pi_{i_{s+1}}^{(c, w)}=\pi_{i_{s+1}}^{(c, u)}-1, \delta^{(c, v)}=\delta^{(c, u)}-\beta_{i_{0}}^{\tau}+\beta_{i_{s+1}}^{\tau}$ and $\delta^{(c, w)}=\delta^{(c, u)}+\beta_{i_{0}}^{\tau^{\prime}}-\beta_{i_{s+1}}^{\tau^{\prime}}$.

If $s$ is even, then $\pi_{i_{s+1}}^{(c, v)}=\pi_{i_{s+1}}^{(c, u)}-1, \pi_{i_{s+1}}^{(c, w)}=\pi_{i_{s+1}}^{(c, u)}+1, \delta^{(c, v)}=\delta^{(c, u)}-\beta_{i_{0}}^{\tau}-$ $\beta_{i_{s+1}}^{k-1-\tau}$ and $\delta^{(c, w)}=\delta^{(c, u)}+\beta_{i_{0}}^{\tau^{\prime}}+\beta_{i_{s+1}}^{k-1-\tau^{\prime}}$.

Therefore $\pi^{(c, u)}=\frac{1}{2}\left(\pi^{(c, v)}+\pi^{(c, w)}\right)$. Moreover, $\pi^{(c, v)} \neq \pi^{(c, u)}$. We will show next that

$$
\frac{1}{2}\left(\delta^{(c, v)}+\delta^{(c, w)}\right) \geq \delta^{(c, u)} .
$$

contradicting the fact that $\pi^{(c, u)} x \geq \delta^{(c, u)}$ is facet-defining.

If $\tau, \tau^{\prime}$ satisfy a), then $\beta_{i_{0}}^{\tau}=0$ and $\beta_{i_{0}}^{\tau^{\prime}}=1$. If $s$ is odd $\delta^{(c, v)} \geq \delta^{(c, u)}$ and $\delta^{(c, w)} \geq \delta^{(c, u)}$, while, if $s$ is even, $\delta^{(c, v)} \geq \delta^{(c, u)}-1$ and $\delta^{(c, w)} \geq \delta^{(c, u)}+1$. In both cases, (9) holds.

If $\tau, \tau^{\prime}$ satisfy b), then $\beta_{i_{0}}^{\tau}=\beta_{i_{0}}^{\tau^{\prime}}$. If $s$ is odd, then $\delta^{(c, v)}+\delta^{(c, w)}=$ $2 \delta^{(c, u)}+\beta_{i_{s+1}}^{\tau}-\beta_{i_{s+1}}^{\tau^{\prime}} \geq 2 \delta^{(c, u)}$, where the inequality follows from the fact that $\beta_{i_{s+1}}^{\tau} \geq \beta_{i_{s+1} \tau^{\prime}}$, since $\tau>\tau^{\prime}$. If $s$ is even, then $\delta^{(c, v)}+\delta^{(c, w)}=2 \delta^{(c, u)}-$ $\beta_{i_{s+1}}^{k-1-\tau}+\beta_{i_{s+1}}^{k-1-\tau^{\prime}} \geq 2 \delta^{(c, u)}$, where the inequality follows from the fact that $\beta_{i_{s+1}}^{k-1-\tau^{\prime}} \geq \beta_{i_{s+1}}^{k-1-\tau}$, since $k-1-\tau^{\prime}>k-1-\tau$.

Remark 20 If the skeleton of a facet-defining inequality $\pi^{(c, u)} x \geq \delta^{(c, u)}$ induces a tree $T$, then by Remark 16, the set of leaves of $T$ is $V(T) \cap I$. In this case, $(c, u)$ is completely determined by $T$ and by the vectors $u_{n(i) i}$, 
$i \in I$. Indeed, let $i$ be a leaf of $T \backslash I$ and $l$ be the unique node of $T \backslash I$ adjacent to $i$, then

$$
c_{i}=\max _{t=0, \ldots, k-1}\left\{\sum_{j \in I: i j \in E} u_{i j}^{t}\right\},
$$

as otherwise $u_{i l}^{t}>0, t=0, \ldots, k-1$, a contradiction to Assumption A5). Since $u_{i l}^{t}=c_{i}-\sum_{j \in I: i j \in E} u_{i j}^{t}$, we can proceed recursively on a leaf of $T \backslash$ $(I \cup\{i\})$, to reconstruct the vector $(c, u)$.

\section{$5 \quad$ On mixing sets}

In this section, we use the results established so far to derive descriptions of the facet-defining inequalities of special types of mixed integer sets.

\subsection{Mixing set}

Recall that the mixing set is the set

$$
X_{n}^{M I X}=\left\{\left(x_{0}, x\right) \in \mathbb{R} \times \mathbb{Z}^{n} \mid x_{0}+x_{i} \geq b_{i}, i=1, \ldots, n\right\} .
$$

When there is no ambiguity, we denote $X_{n}^{M I X}$ simply by $X^{M I X}$. The facetdefining inequalities describing the convex hull of $X^{M I X}$ have been characterized by Günlük and Pochet [5]. These are the so called mixing inequalities that we now define. Let $f_{i}=b_{i}-\left\lfloor b_{i}\right\rfloor$, and assume that $f_{1} \geq \ldots \geq f_{n} \geq 0$. Given any sequence $1 \leq i_{1}<\ldots<i_{m} \leq n$. The mixing inequality relative to this sequence is

$$
x_{0}+\sum_{h=1}^{m-1}\left(f_{i_{h}}-f_{i_{h+1}}\right)\left(x_{i_{h}}-\left\lfloor b_{i_{h}}\right\rfloor\right)+\left(1-f_{i_{1}}+f_{i_{m}}\right)\left(x_{m}-\left\lfloor b_{i_{m}}\right\rfloor\right) \geq f_{i_{1}} .
$$

Theorem 21 conv $\left(X^{M I X}\right)$ is defined by the mixing inequalities.

Proof: By the usual change of variables $x_{i}^{\prime}=x_{i}-\left\lfloor b_{i}\right\rfloor$, we may assume $b_{i}=f_{i}=h_{i} / k$ for some integers $k$ and $h_{i}, i=1, \ldots, n$. The extended formulation for $X^{M I X}$, represented in Figure 2, becomes

(c) $k x_{0}-\mu_{0}^{0}-\cdots-\mu_{0}^{k-1}=0$,

$\left(u_{i}^{t}\right) \quad \mu_{0}^{t}+x_{i} \geq 0, t=0, \ldots, k-h_{i}-1, i=1, \ldots, n ;$

$\left(u_{i}^{t}\right) \quad \mu_{0}^{t}+x_{i} \geq 1, \quad t=k-h_{i}, \ldots, k-1, i=1, \ldots, n$.

Let $(c, u)$ be a vector satisfying (6), namely $\sum_{i=1}^{n} u_{i}^{t}=c, t=0, \ldots, k-1$, $u \geq 0$, such that $\pi^{(c, u)} x \geq \delta^{(c, u)}$ is facet-defining for $\operatorname{conv}\left(X^{M I X}\right)$. Then we may assume that $(c, u)$ satisfy A1), ., A5). By Lemma 18, we may also assume $h_{1}>\ldots>h_{n}$. Notice also that $c=1$. Otherwise, if $c>1$, for $t=0, \ldots, k-1$, choose $i(t)$ such that $u_{i(t)}^{t}>0$. Define $u_{i(t)}^{\prime t}=1$, 
$t=0, \ldots, k-1$, and ${u^{\prime}}_{i}^{t}=u_{i}^{t}$ otherwise. Let $c^{\prime}=1,\left(c^{\prime \prime}, u^{\prime \prime}\right)=(c, u)-\left(c^{\prime}, u^{\prime}\right)$. Then $\left(c^{\prime}, u^{\prime}\right),\left(c^{\prime \prime}, u^{\prime \prime}\right)$ satisfy $(6)$ and $(c, u)=\left(c^{\prime}, u^{\prime}\right)+\left(c^{\prime \prime}, u^{\prime \prime}\right)$. By Remark 15, $\pi^{(c, u)} x \geq \delta^{(c, u)}$ is not facet-defining, a contradiction. Therefore $c=1$ and $u$ has 0,1 components. Since $\sum_{i=1}^{n} u_{i}^{t}=1, t=0, \ldots, k-1$, it follows from Lemma 19 that the vector $u_{i}$ is the vector defined by $u_{i}^{t}=1$ for $t=k-h_{i}, \ldots, k-h_{i+1}-1$ (the indices being taken modulo $k$ ), $u_{i}^{t}=0$ otherwise. The inequality $\pi^{(u, v)} x \geq \delta^{(u, v)}$ is therefore

$$
k x_{0}+\sum_{i=1}^{n-1}\left(h_{i}-h_{i+1}\right) x_{i}+\left(h_{n}+k-h_{1}\right) x_{n} \geq \underbrace{\sum_{h=1}^{n-1}\left(h_{i}-h_{i+1}\right)+h_{n}}_{=h_{1}}
$$

Dividing the inequality by $k$ gives the mixing inequality.

We should point out that in the literature $[5,8]$, the following mixed integer set has been usually studied

$$
X^{M I X_{+}}=\left\{\left(x_{0}, x\right) \in \mathbb{R}_{+} \times \mathbb{Z}^{n} \mid x_{0}+x_{i} \geq b_{i}, i=1, \ldots, n\right\} .
$$

If we define $b_{n+1}=0, X^{M I X_{+}}$is the set of points $\left(x_{0}, x\right)$ such that $\left(x_{0}, x, 0\right)$ is in $\left\{\left(x_{0}, x, x_{n+1}\right) \in \mathbb{R} \times \mathbb{Z}^{n+1} \mid x_{0}+x_{i} \geq b_{i}, i=1, \ldots, n+1\right\}$. Notice that the latter is a mixing set $X_{n+1}^{M I X}$. Therefore $X^{M I X_{+}}$is the projection on the space of variables $x_{0}, \ldots, x_{n}$ of $X_{n+1}^{M I X} \cap\left\{\left(x_{0}, x, x_{n+1}\right) \mid x_{n+1}=\right.$ $0\}$. By Proposition 8, the convex hull of the latter set is $\operatorname{conv}\left(X_{n+1}^{M I X}\right) \cap$ $\left\{\left(x_{0}, x, x_{n+1}\right) \mid x_{n+1}=0\right\}$. Therefore the irredundant inequalities for $X^{M I X_{+}}$ are obtained from the mixing inequalities for $X_{n+1}^{M I X}$ by setting $x_{n+1}$ to 0 . Given any sequence $1 \leq i_{1}<\ldots<i_{m} \leq n$, there are two mixing inequalities associated with it, the mixing inequality (11) and the one obtained by the mixing inequality for $X_{n+1}^{M I X}$ relative to the sequence $1 \leq i_{1}<\ldots<i_{m}<$ $i_{m+1}=n+1$ by setting $x_{n+1}$ to 0 , namely

$$
x_{0}+\sum_{h=1}^{m}\left(f_{i_{h}}-f_{i_{h+1}}\right)\left(x_{i_{h}}-\left\lfloor b_{i_{h}}\right\rfloor\right) \geq f_{i_{1}} .
$$

\subsection{Double mixing set}

Here we consider the double mixing set

$$
X^{G M I X}=\left\{\left(x_{0}, x\right) \in \mathbb{R} \times \mathbb{Z}^{n} \mid b_{i} \leq x_{0}+x_{i} \leq d_{i}, i=1, \ldots, n\right\} .
$$

We let

$$
\begin{gathered}
X^{M I X \geq}=\left\{\left(x_{0}, x\right) \in \mathbb{R} \times \mathbb{Z}^{n} \mid x_{0}+x_{i} \geq b_{i}, i=1, \ldots, n\right\}, \\
X^{M I X \leq}=\left\{\left(x_{0}, x\right) \in \mathbb{R} \times \mathbb{Z}^{n} \mid-x_{0}-x_{i} \geq-d_{i}, i=1, \ldots, n\right\} .
\end{gathered}
$$

These last two sets are mixing sets, thus their facet-defining inequalities are described in Theorem 21. 
Theorem $22 \operatorname{conv}\left(X^{G M I X}\right)$ is the set of points $x \in \mathbb{R}^{1+n}$ satisfying

$$
\begin{gathered}
x_{i}-x_{j} \geq\left\lceil b_{i}-d_{j}\right\rceil, \quad 1 \leq i, j \leq n, \\
x \in \operatorname{conv}\left(X^{M I X \geq}\right) \cap \operatorname{conv}\left(X^{M I X \leq}\right) .
\end{gathered}
$$

Proof: There exist integers $k, h_{i}, l_{i}, i=1, \ldots, n$, such that $b_{i}=\left\lfloor b_{i}\right\rfloor+h_{i} / k$, $d_{i}=\left\lfloor d_{i}\right\rfloor+l_{i} / k$.

The extended formulation for $X^{G M I X}$ is

$$
\begin{aligned}
k x_{0}-\mu_{0}^{0}-\cdots-\mu_{0}^{k-1} & =0, \\
\mu_{0}^{t}+x_{i} & \geq\left\lfloor b_{i}\right\rfloor, \quad t=0, \ldots, k-h_{i}-1, i=1, \ldots, n ; \\
\mu_{0}^{t}+x_{i} & \geq\left\lceil b_{i}\right\rceil, \quad t=k-h_{i}, \ldots, k-1, i=1, \ldots, n ; \\
-\mu_{0}^{t}-x_{i} & \geq\left\lceil-d_{i}\right\rceil, \quad t=0, \ldots, k-l_{i}-1, i=1, \ldots, n ; \\
-\mu_{0}^{t}-x_{i} & \geq\left\lfloor-d_{i}\right\rfloor, \quad t=k-l_{i}, \ldots, k-1, i=1, \ldots, n .
\end{aligned}
$$

Any valid inequality for $X^{G M I X}$ is of the form $\rho^{(c, u, v)} x \geq \gamma^{(c, u, v)}$, where

$$
\begin{aligned}
\rho^{(c, u, v)} x & =k c x_{0}+\sum_{i=1}^{n} \sum_{t=0}^{k-1}\left(u_{i}^{t}-v_{i}^{t}\right) x_{i} \\
\gamma^{(c, u, v)} & =\sum_{i=1}^{n}\left(\sum_{t=0}^{k-h_{i}-1} u_{i}^{t}\left\lfloor b_{i}\right\rfloor+\sum_{t=k-h_{i}}^{k-1} u_{i}^{t}\left\lceil b_{i}\right\rceil+\sum_{t=0}^{k-l_{i}-1} v_{i}^{t}\left\lceil-d_{i}\right\rceil+\sum_{t=k-l_{i}}^{k-1} v_{i}^{t}\left\lfloor-d_{i}\right\rfloor\right)
\end{aligned}
$$

where

$$
\sum_{i=1}^{n}\left(u_{i}^{t}-v_{i}^{t}\right)=c \text { for } t=0, \ldots, k-1, \quad u, v \geq 0 .
$$

Notice that, if $v=0$, then $\rho^{(c, u, v)} x \geq \gamma^{(c, u, v)}$ is valid for $X^{M I X \geq}$, while if $u=$ 0 , then $\rho^{(c, u, v)} x \geq \gamma^{(c, u, v)}$ is valid for $X^{M I X \leq}$. Therefore we are interested in the facet-defining inequalities for which $u \neq 0, v \neq 0$. We assume $(c, u, v)$ is integer with coprime entries. In this case, we show that $c=0$. If not, then by symmetry assume $c>0$. For $t=0, \ldots, k-1$, choose $i(t)$ such that $u_{i(t)}^{t}>0$. Define $u_{i(t)}^{\prime t}=1, t=0, \ldots, k-1$, and $u_{i}^{\prime t}=u_{i}^{t}$ otherwise. Let $c^{\prime}=1,\left(c^{\prime \prime}, u^{\prime \prime}\right)=(c, u)-\left(c^{\prime}, u^{\prime}\right)$. Then $\left(c^{\prime}, u^{\prime}, 0\right),\left(c^{\prime \prime}, u^{\prime \prime}, v\right)$ satisfy (13) and $(c, u, v)=\left(c^{\prime}, u^{\prime}, 0\right)+\left(c^{\prime \prime}, u^{\prime \prime}, v\right)$. By Remark 15, $\rho^{(c, u, v)} x \geq \gamma^{(c, u, v)}$ is not facet-defining, a contradiction.

Thus $\sum_{i=1}^{n} u_{i}^{t}=\sum_{i=1}^{n} v_{i}^{t}$ for $t=0, \ldots, k-1$. Next we show that, for some $\bar{t} \in\{0, \ldots, k-1\}$ and some $i, j \in\{1, \ldots, n\}, u_{i}^{\bar{t}}=v_{j}^{\bar{t}}=1$, and $u$, $v$ are zero everywhere else. If not, choose $\bar{t} \in\{0, \ldots, k-1\}$ such that $i, j \in\{1, \ldots, n\} u_{i}^{\bar{t}}>0, v_{j}^{\bar{t}}>0$. Define $u^{\prime}, v^{\prime}$ by $u_{i}^{\prime \bar{t}}=v_{j}^{\prime \bar{t}}=1, u^{\prime}, v^{\prime}$ be zero in all other entries. Let $u^{\prime \prime}=u-u^{\prime}, v^{\prime \prime}=v-v^{\prime}$. Then $\left(0, u^{\prime}, v^{\prime}\right),\left(0, u^{\prime \prime}, v^{\prime \prime}\right)$ both satisfy $(13)$, and $(0, u, v)=\left(0, u^{\prime}, v^{\prime}\right)+\left(0, u^{\prime \prime}, v^{\prime \prime}\right)$. By Remark 15, $\rho^{(0, u, v)} x \geq \gamma^{(0, u, v)}$ is not facet-defining, a contradiction.

Therefore $\rho^{(c, u, v)} x=x_{i}-x_{j}$, and $i \neq j$. We only need to show $\gamma^{(c, u, v)}=$ $\left\lceil b_{i}-d_{j}\right\rceil$. If one among $b_{i}$ and $d_{j}$ is integer, then it is not difficult to see 
that the optimal choice of $\bar{t}$ gives $\gamma^{(c, u, v)}=\left\lceil b_{i}\right\rceil+\left\lceil-d_{j}\right\rceil=\left\lceil b_{i}-d_{j}\right\rceil$. Thus we assume $b_{i}-\left\lfloor b_{i}\right\rfloor>0,-d_{j}-\left\lfloor-d_{j}\right\rfloor>0$. If $b_{i}-\left\lfloor b_{i}\right\rfloor+\left(-d_{j}-\left\lfloor-d_{j}\right\rfloor\right) \leq 1$, then $k-l_{j} \leq k-h_{i}$, therefore the optimal choice of $\bar{t}$ is either $\bar{t} \leq k-l_{j}-1$ or $\bar{t} \geq k-h_{i}$, which gives $\gamma^{(c, u, v)}=\left\lfloor b_{i}\right\rfloor+\left\lceil-d_{j}\right\rceil=\left\lceil b_{i}-d_{j}\right\rceil$. Finally, if $b_{i}-\left\lfloor b_{i}\right\rfloor+\left(-d_{j}-\left\lfloor-d_{j}\right\rfloor\right)>1$, then $k-l_{j}>k-h_{i}$, therefore the optimal choice of $\bar{t}$ is $k-h_{i} \leq \bar{t} \leq k-l_{j}-1$, which gives $\gamma^{(c, u, v)}=\left\lceil b_{i}\right\rceil+\left\lceil-d_{j}\right\rceil=\left\lceil b_{i}-d_{j}\right\rceil$.

\subsection{Continuous mixing set with flows}

The continuous mixing set with flows is the following:

$X_{n}^{C M F}=\left\{(s, r, z, x) \in \mathbb{R} \times \mathbb{R}_{+}^{n} \times \mathbb{R}_{+}^{n} \times \mathbb{Z}^{n} \mid s+r_{i}+z_{i} \geq b_{i}, z_{i} \leq x_{i}, i=1, \ldots, n\right\}$.

When there is no ambiguity, we denote $X_{n}^{C M F}$ simply by $X^{C M F}$. We describe the facet-defining inequalities for $\operatorname{conv}\left(X^{C M F}\right)$ in the original space. We will discuss later how this model encompasses the continuous mixing set of Van Vyve [13]. Conforti, Di Summa, and Wolsey [3] give a polynomial size extended formulation for the convex hull of $X^{C M F_{+}}=$ $X^{C M F} \cap\{(s, r, z, x) \mid s \geq 0\}$, but describing the facet-defining inequalities for $\operatorname{conv}\left(X^{C M F_{+}}\right)$in the original space was an open problem. We will show how the description for $\operatorname{conv}\left(X^{C M F_{+}}\right)$can be derived from that for $\operatorname{conv}\left(X^{C M F}\right)$.

Let $f_{i}=b_{i}-\left\lfloor b_{i}\right\rfloor$, and assume that $f_{1} \geq \ldots \geq f_{n} \geq 0$. Every facet for $\operatorname{conv}\left(X^{C M F}\right)$ is of the following form. Let $(X, T)$ be a partition of $V(G)$. Consider the complete directed graph $D$ on $n$ nodes, and let $C$ be a directed cycle of $D$. Let $\gamma_{C}$ denote the number of arcs $(i, j)$ in $C$ such that $i>j$. The following is the cycle inequality associated with the cycle $C$ and the partition $(X, T)$.

$$
\begin{gathered}
\gamma_{C} s+\sum_{i \in V(C)} r_{i}+\sum_{i \in T \cap V(C)} z_{i}+\sum_{\substack{(i, j) \in C \\
i<j, i \in X}}\left(f_{i}-f_{j}\right) x_{i}+\sum_{\substack{(i, j) \in C \\
i>j, i \in X}}\left(1+f_{i}-f_{j}\right) x_{i} \geq \\
\sum_{\substack{(i, j) \in C \\
i>j}} f_{j}+\sum_{\substack{(i, j) \in C \\
i<j}}\left(f_{i}-f_{j}\right)\left\lfloor b_{i}\right\rfloor+\sum_{\substack{(i, j) \in C \\
i>j}}\left(1+f_{i}-f_{j}\right)\left\lfloor b_{i}\right\rfloor .
\end{gathered}
$$

Given a partition $(X, T)$ of $V(G)$, let

$$
X^{(X, T)}=\left\{(s, r, z, x) \in \mathbb{R} \times \mathbb{R}_{+}^{n} \times \mathbb{R}^{n} \times \mathbb{Z}^{n} \mid \begin{array}{ll}
s+r_{i}+z_{i} \geq b_{i} & i=1, \ldots, n \\
z_{i} \geq 0 & i \in T \\
z_{i} \leq x_{i} & i \in X
\end{array}\right\} .
$$

Theorem 23 Given $b_{1}, \ldots, b_{n} \in \mathbb{R}$ such that $f_{1} \geq f_{2} \geq \ldots \geq f_{n}$, we have

$$
\operatorname{conv}\left(X^{C M F}\right)=\bigcap \operatorname{conv}\left(X^{(X, T)}\right),
$$


where the intersection ranges over all partitions $(X, T)$ of $V(G)$.

Furthermore, for a given partition $(X, T)$ of $V(G), \operatorname{conv}\left(X^{(X, T)}\right)$ is defined by the inequalities $s+r_{i}+z_{i} \geq b_{i}, r_{i} \geq 0, i=1, \ldots, n, z_{i} \geq 0, i \in T, z_{i} \leq x_{i}$, $i \in X$, and by the cycle inequalities (14) for every directed cycle $C$.

Proof: We can transform $X^{C M F}$ into a set of the usual form by applying the change of variables $y_{i}=s+r_{i}, z_{i}^{\prime}=z_{i}-b_{i}, x_{i}^{\prime}=x_{i}-\left\lfloor b_{i}\right\rfloor, i=1, \ldots, n$. The inequalities $r_{i} \geq 0$ become

$$
-s+y_{i} \geq 0
$$

the inequalities $s+r_{i}+z_{i} \geq b_{i}$ become

$$
y_{i}+z_{i}^{\prime} \geq 0
$$

the inequalities $-z_{i}+x_{i} \geq 0$ become

$$
-z_{i}^{\prime}+x_{i}^{\prime} \geq f_{i}
$$

while the inequalities $z_{i} \geq 0$ become

$$
z_{i}^{\prime}+\ell_{i} \geq-f_{i}
$$

and

$$
\ell_{i}=\left\lfloor b_{i}\right\rfloor
$$

Consider the sets

$$
\begin{aligned}
& S=\left\{\left(s, y, z^{\prime}, x^{\prime}, \ell\right) \text { satisfying (16)-(19) } i=1, \ldots, n, x^{\prime}, \ell \in \mathbb{Z}^{n}\right\} \\
& S^{(X, T)}=\left\{\begin{array}{ll}
(16)-(17) i=1, \ldots, n, \\
\left(s, y, z^{\prime}, x^{\prime}, \ell\right) \text { satisfying } \begin{array}{l}
(18) i \in X,(19) i \in T, \\
x^{\prime}, \ell \in \mathbb{Z}^{n}
\end{array}
\end{array}\right\}
\end{aligned}
$$

The continuous mixing set with flows $X^{C M F}$ is equivalent to $S \cap\left\{\left(s, y, z^{\prime}, x^{\prime}, \ell\right) \mid \ell_{i}=\right.$ $\left.\left\lfloor b_{i}\right\rfloor, i=1, \ldots, m\right\}$, while $X^{(X, T)}$ is equivalent to $S^{(X, T)} \cap\left\{\left(s, y, z^{\prime}, x^{\prime}, \ell\right) \mid \ell_{i}=\right.$ $\left.\left\lfloor b_{i}\right\rfloor, i=1, \ldots, m\right\}$.

Let $G$ be the graph whose nodes are the variables $s, y_{i}, z_{i}^{\prime}, x_{i}^{\prime}$ and $\ell_{i}$, $i=1, \ldots, n$, and where two variables are joined by an edge when they appear in one of the inequalities (16)-(17). Note that $G$ is a tree, thus $S$ and $S^{(X, T)}$ fit the framework of Section 4.1.

As usual, let $k, h_{i}, i=1, \ldots, n$ be integers, such that $f_{i}=h_{i} / k$. The 
extended formulation for $\operatorname{conv}(S)$ is

$$
\begin{array}{rrll}
(c) & -k s+\mu_{0}^{0}+\cdots+\mu_{0}^{k-1} & =0, & \\
\left(p_{i}\right) & k y_{i}-\mu_{i}^{0}-\cdots-\mu_{i}^{k-1} & =0, & i=1, \ldots, n \\
\left(q_{i}\right) & k z_{i}^{\prime}-\sigma_{i}^{0}-\cdots-\sigma_{i}^{k-1} & =0, & i=1, \ldots, n \\
\left(v_{i}^{t}\right) & -\mu_{0}^{t}+\mu_{i}^{t} & \geq 0, & t=0, \ldots, k-1, i=1, \ldots, n ; \\
\left(u_{i}^{t}\right) & \mu_{i}^{t}+\sigma_{i}^{k-1-t} & \geq 0, & t=0, \ldots, k-1, i=1, \ldots, n ; \\
\left(w_{i}^{t}\right) & -\sigma_{i}^{t}+x_{i}^{\prime} & \geq 1, & t=0, \ldots, h_{i}-1, i=1, \ldots, n ; \\
\left(w_{i}^{t}\right) & -\sigma_{i}^{t}+x_{i}^{\prime} & \geq 0, & t=h_{i}, \ldots, k-1, i=1, \ldots, n ; \\
\left(m_{i}^{t}\right) & \sigma_{i}^{t}+\ell_{i} & \geq-1, & t=0, \ldots, h_{i}-1, i=1, \ldots, n ; \\
\left(m_{i}^{t}\right) & \sigma_{i}^{t}+\ell_{i} & \geq 0, & t=h_{i}, \ldots, k-1, i=1, \ldots, n .
\end{array}
$$

Any valid inequality for $\operatorname{conv}(S)$ is thus of the form

$$
-k c s+\sum_{i=1}^{n} k p_{i} y_{i}+\sum_{i=1}^{n} k q_{i} z_{i}+\sum_{i=1}^{n}\left(\sum_{t=0}^{k-1} w_{i}^{t}\right) x_{i}^{\prime}+\sum_{i=1}^{n}\left(\sum_{t=0}^{k-1} m_{i}^{t}\right) \ell_{i} \geq \sum_{i=1}^{n}\left(\sum_{t=0}^{h_{i}-1} w_{i}^{t}-\sum_{t=0}^{h_{i}-1} m_{i}^{t}\right)
$$

where $(c, p, q, v, u, w, m)$ satisfy

$$
\begin{aligned}
\sum_{i=1}^{n} v_{i}^{t} & =c \quad t=0, \ldots, k-1 \\
v_{i}^{t}+u_{i}^{t} & =p_{i} \quad t=0, \ldots, k-1, \quad i=1, \ldots, n \\
u_{i}^{k-1-t}-w_{i}^{t}+m_{i}^{t} & =q_{i} \quad t=0, \ldots, k-1, \quad i=1, \ldots, n
\end{aligned}
$$

Note that, given a partition $(X, T)$ of $V(G)$, any valid inequality for $\operatorname{conv}\left(S^{(X, T)}\right)$ is of the form (20), where $(c, p, q, v, u, w, m)$ satisfy (21), and such that $w_{i}^{t}=0$ for every $i \in T, t=0, \ldots, k-1$ and $m_{i}^{t}=0$ for every $i \in X$, $t=0, \ldots, k-1$.

The rest of the proof is divided into two parts. In part I we show that (15) holds, while in part II we show that any nontrivial inequality for $\operatorname{conv}\left(X^{(X, T)}\right)$ is a cycle inequality.

I. Since $X^{(X, T)}$ contains $X^{C M F}$, the inclusion " $\subseteq$ " of $(15)$ is trivial. We show the reverse inclusion.

We will show that, given an inequality of the form (20), where $(c, p, q, v, u, w, m)$ satisfy (21), if such an inequality is facet-defining for $\operatorname{conv}(S)$, then there exists a partition $(X, T)$ of $V(G)$ such that $w_{i}^{t}=0$ for every $i \in T, t=$ $0, \ldots, k-1$ and $m_{i}^{t}=0$ for every $i \in X, t=0, \ldots, k-1$. This implies that the inequality is valid for $\operatorname{conv}\left(S^{(X, T)}\right)$.

As usual, we assume that $(c, p, q, v, u, w, m)$ has entries that are integer and coprime, and that the inequality (20) is facet-defining. Notice that, even though the inequalities defining the set $S$ contain terms with negative coefficients, assumption A5) still holds, as one can verify using the transformation in Proposition 10. 
Claim 1 The following chains of inequalities hold.

$$
\begin{aligned}
& w_{i}^{h_{i}-1} \geq w_{i}^{h_{i}-2} \geq \ldots \geq w_{i}^{0} \geq w_{i}^{k-1} \geq \ldots \geq w_{i}^{h_{i}} ; \\
& m_{i}^{h_{i}} \geq m_{i}^{h_{i}+1} \geq \ldots \geq m_{i}^{k-1} \geq m_{i}^{0} \geq \ldots \geq m_{i}^{h_{i}-1} \\
& u_{i}^{k-h_{i}} \geq u_{i}^{k-h_{i}+1} \geq \ldots \geq u_{i}^{k-1} \geq u_{i}^{0} \geq \ldots \geq u_{i}^{k-h_{i}-1} .
\end{aligned}
$$

To prove (22),(23), we will apply Lemma 19. We thus need to apply the transformation in Proposition 10 to turn our problem into one of the form $S^{(G, I)}$. We introduce variables $z_{i}^{\prime \prime}, i=1, \ldots, n$, and replace the inequality $-z_{i}^{\prime}+x_{i} \geq f_{i}$ by $z_{i}^{\prime}+z_{i}^{\prime \prime}=0$ and $z_{i}^{\prime \prime}+x_{i} \geq f_{i}, i=1, \ldots, n$. In the extended formulation, we have integer variables $\sigma_{i}^{\prime 0}, \ldots, \sigma_{i}^{\prime k-1}$, where $z_{i}^{\prime \prime}=$ $\sigma_{i}^{\prime 0}+\cdots+\sigma_{i}^{\prime k-1}$. The inequalities relative to $z_{i}^{\prime}+z_{i}^{\prime \prime}=0$ in the extended formulation are $\sigma_{i}^{t}+\sigma_{i}^{\prime k-1-t}=0, t=1, \ldots, k-1, i=1, \ldots, n$. The inequalities relative to $z_{i}^{\prime \prime}+x_{i} \geq f_{i}$, are $\sigma_{i}^{\prime t}+x_{i} \geq 0$, for $0 \leq t \leq k-h_{i}-1$, $\sigma_{i}^{\prime t}+x_{i} \geq 1$, for $k-h_{i} \leq t \leq k-1$, and we associate projection multipliers $w_{i}^{\prime t}$ to such inequalities, $0 \leq t \leq k-1, i=1, \ldots, n$. Since $\sigma_{i}^{t}=-\sigma^{k-t-1}$, we have that $w_{i}^{t}=w_{i}^{\prime k-t-1}, 0 \leq t \leq k-1, i=1, \ldots, n$. By Lemma 19,

$$
\begin{aligned}
& w_{i}^{\prime k-h_{i}} \geq w_{i}^{\prime k-h_{i}+1} \geq \ldots \geq w_{i}^{\prime k-1} \geq w_{i}^{\prime 0} \geq \ldots \geq w_{i}^{\prime k-h_{i}-1} \\
& m_{i}^{h_{i}} \geq m_{i}^{h_{i}+1} \geq \ldots \geq m_{i}^{k-1} \geq m_{i}^{0} \geq \ldots \geq m_{i}^{h_{i}-1}
\end{aligned}
$$

and $(22),(23)$ follow.

Finally, (24) follows from (22),(23) and the third equation in (21).

Claim 2 For $i=0, \ldots, n$ and $t=0, \ldots, k-1, m_{i}^{t}=0$ or $w_{i}^{t}=0$.

Indeed, assume that both $m_{i}^{t}>0$ and $w_{i}^{t}>0$ for some $i$ and $t$. Let $\left(c^{\prime}, p^{\prime}, q^{\prime}, v^{\prime}, u^{\prime}, w^{\prime}, m^{\prime}\right)$ be obtained from $(c, p, q, v, u, w, m)$ by decreasing $m_{i}^{t}$ and $w_{i}^{t}$ by 1 and leaving all other components unchanged. Let $\left(c^{\prime \prime}, p^{\prime \prime}, q^{\prime \prime}, v^{\prime \prime}, u^{\prime \prime}, w^{\prime \prime}, m^{\prime \prime}\right)$ be the vector having all components equal to 0 , except $m_{i}^{t}=w_{i}^{t}=1$. Then $\left(c^{\prime}, p^{\prime}, q^{\prime}, v^{\prime}, w^{\prime}, m^{\prime}\right)$ and $\left(c^{\prime \prime}, p^{\prime \prime}, q^{\prime \prime}, v^{\prime \prime}, u^{\prime \prime}, w^{\prime \prime}, m^{\prime \prime}\right)$ satisfy $(21)$, and $(c, p, q, v, u, w, m)=$ $\left(c^{\prime}, p^{\prime}, q^{\prime}, v^{\prime}, u^{\prime}, w^{\prime}, m^{\prime}\right)+\left(c^{\prime \prime}, p^{\prime \prime}, q^{\prime \prime}, v^{\prime \prime}, u^{\prime \prime}, w^{\prime \prime}, m^{\prime \prime}\right)$. By Remark 15, the inequality $(20)$ defined by $(c, p, q, v, u, w, m)$ is not facet-defining for $\operatorname{conv}(S)$, a contradiction. This concludes the proof of the claim.

Claim 3 For $i=1, \ldots, n, q_{i} \geq 0$. If $q_{i}>0$, then $w_{i}^{t}=0,0 \leq t \leq k-1$. If $q_{i}=0$, then $m_{i}^{t}=0,0 \leq t \leq k-1$.

If $q_{i}<0$, then by $(21), w_{i}^{t}>0, t=0, \ldots, k-1$, a contradiction to Assumption A5). Hence $q_{i} \geq 0$

By Remark 20

$$
q_{i}=\max _{t=0, \ldots, k-1}\left\{m_{i}^{t}-w_{i}^{t}\right\}
$$


Assume $q_{i}=0$. If $m_{i}^{t}>0$ for some $t$, then by (25) $w_{i}^{t}>0$, a contradiction to Claim 2. Therefore $m_{i}^{t}=0, t=0, \ldots, k-1$.

Finally, assume $q_{i}>0$. If $w_{i}^{t}>0$, then by $(25) m_{i}^{t}>0$, again a contradiction to Claim 2. Therefore $w_{i}^{t}=0, t=0, \ldots, k-1$. This concludes the proof of the claim.

We define the set $X^{\prime}$ as the set of indices $i$ in $\{1, \ldots, n\}$ such that $w_{i}^{t}>0$ for some $t, 0 \leq t \leq k-1$, and $T^{\prime}$ the set of indices $i$ in $\{1, \ldots, n\}$ such that $m_{i}^{t}>0$ for some $t, 0 \leq t \leq k-1$. By Claim $3, X^{\prime}$ and $T^{\prime}$ are disjoint, so there exists a partition $(X, T)$ of $V(G)$ such that $X^{\prime} \subseteq X$ and $T^{\prime} \subseteq T$. Furthermore, Claims 2 and 3 imply that $m_{i}^{t}=0, t=0, \ldots, k-1$, for every $i \in X$, while $w_{i}^{t}=0, t=0, \ldots, k-1$, for every $i \in T$.

II Let $(X, T)$ be a partition of $V(G)$.

We first show that the cycle inequalities are valid for $\operatorname{conv}\left(X^{(X, T)}\right)$. Given a cycle $C$ in $D$, we describe the multipliers $(\bar{c}, \bar{p}, \bar{q}, \bar{v}, \bar{u}, \bar{w}, \bar{m})$ in (20) that determine the cycle inequality associated to $C$ and $(X, T)$. We define vector $\bar{u}$ as follows.

- For every $\operatorname{arc}(i, j)$ in $C$, if $i<j$ we let $\bar{u}_{i}^{t}=1$ for $t \in\left\{k-h_{i}, \ldots, k-h_{j}-1\right\}$, 0 otherwise;

-For every $\operatorname{arc}(i, j)$ in $C$, if $i>j$, we let $\bar{u}_{i}^{t}=0$ for $t \in\left\{k-h_{j}, \ldots, k-h_{i}-1\right\}$, 1 otherwise;

- For every $i \notin V(C)$, we let $\bar{u}_{i}^{t}=0, t=0, \ldots, k-1$.

We define $\bar{p}_{i}=1$ for $i \in V(C), \bar{p}_{i}=0$ otherwise. Thus, by (21), for $i=1, \ldots, n$ and $t=0, \ldots, k-1, \bar{v}_{i}^{t}=1-\bar{u}_{i}^{t}$ if $i \in V(C), \bar{v}_{i}^{t}=0$ for $i \notin V(C)$.

One can verify that, since $C$ is a cycle, $\sum_{i=1}^{n} \bar{v}_{i}^{t}=\sum_{i=1}^{n} \bar{v}_{i}^{t^{\prime}}$ for $0 \leq t \leq$ $t^{\prime} \leq k-1$. Let $\bar{c}=\sum_{i=1}^{n} \bar{v}_{i}^{t}$ for any $t \in\{0, \ldots, k-1\}$. Note that, for $(i, j) \in C, \bar{u}_{i}^{0}=0$ if $i<j$, while $\bar{u}_{i}^{0}=1$ if $i>j$. Thus, for $(i, j) \in C, \bar{v}_{i}^{0}=1$ if $i<j$, while $\bar{v}_{i}^{0}=0$ if $i>j$. In particular, $\bar{c}=\sum_{i=1}^{n} \bar{v}_{i}^{0}$ is precisely the number of $\operatorname{arcs}(i, j) \in C$ such that $i<j$, that is $\bar{c}=|C|-\gamma_{C}$.

For every $i \in X$, let $\bar{w}_{i}^{t}=\bar{u}_{i}^{k-1-t}$ and $\bar{m}_{i}^{t}=0, t=0, \ldots, k-1$. Thus $\bar{q}_{i}=0$ for every $i \in X$. For every $i \in T$, let $\bar{w}_{i}^{t}=0$ and $\bar{m}_{i}^{t}=1-\bar{u}_{i}^{k-1-t}$, $t=0, \ldots, k-1$. Thus $\bar{q}_{i}=1$ for every $i \in T$. 
The right-hand-side in (20) is therefore

$$
\begin{aligned}
\sum_{i=1}^{n}\left(\sum_{t=0}^{h_{i}-1} w_{i}^{t}-\sum_{t=0}^{h_{i}-1} m_{i}^{t}\right) & =\sum_{\substack{(i, j) \in C \\
i<j, i \in X}}\left(h_{i}-h_{j}\right)+\sum_{\substack{(i, j) \in C \\
i<j, i \in T}}\left(-h_{j}\right)+\sum_{\substack{(i, j) \in C \\
i>j, i \in X}} h_{i} \\
& =\sum_{\substack{i, j) \in C \\
i<j}}\left(h_{i}-h_{j}\right)+\sum_{\substack{(i, j) \in C \\
i>j}} h_{i}-\sum_{i \in T} h_{i} \\
& =\sum_{\substack{i, j) \in C \\
i>j}} h_{j}-\sum_{i \in T} h_{i} .
\end{aligned}
$$

The inequality obtained with this choice of multipliers is

$$
\begin{gathered}
k\left(\gamma_{C}-|C|\right) s+\sum_{i \in V(C)} k y_{i}+\sum_{i \in T \cap V(C)} k z_{i}^{\prime}+\sum_{\substack{(i, j) \in C \\
i<j, i \in X}}\left(h_{i}-h_{j}\right) x_{i}^{\prime}+\sum_{\substack{(i, j) \in C \\
i>j, i \in X}}\left(k+h_{i}-h_{j}\right) x_{i}^{\prime}+ \\
+\sum_{\substack{(i, j) \in C \\
i<j, i \in T}}\left(k-h_{i}+h_{j}\right) \ell_{i}+\sum_{\substack{(i, j) \in C \\
i>j, i \in T}}\left(h_{j}-h_{i}\right) \ell_{i} \geq \sum_{\substack{(i, j) \in C \\
i>j}} h_{j}-\sum_{i \in T} h_{i} .
\end{gathered}
$$

Note that, dividing (26) by $k$, and replacing $y_{i}$ with $s+r_{i}, z_{i}^{\prime}$ with $z_{i}-b_{i}$, $x_{i}^{\prime}$ with $x_{i}-\left\lfloor b_{i}\right\rfloor$, and setting $\ell_{i}=\left\lfloor b_{i}\right\rfloor, i=1, \ldots, n$, one obtains the cycle inequality (14).

We finally show that any facet-defining inequality for $\operatorname{conv}\left(S^{(X, T)}\right)$ is of the form (26).

Let $(c, p, q, v, u, w, m)$ satisfy $(21), w_{i}^{t}=0$ for every $i \in T, t=0, \ldots, k-$ 1 , and $m_{i}^{t}=0$ for every $i \in X, t=0, \ldots, k-1$. We assume that the inequality (20) associated with multipliers $(c, p, q, v, u, w, m)$ is facet-defining for $\operatorname{conv}\left(S^{(X, T)}\right)$, and that the entries of $(c, p, q, v, u, w, m)$ are integer and coprime.

Claims 2 and 3 imply that $w_{i}^{t}=u_{i}^{k-1-t}, t=0, \ldots, k-1$ for every $i \in X$, while $m_{i}^{t}=q_{i}-u_{i}^{k-1-t}, t=0, \ldots, k-1$ for every $i \in T$.

Given $j=1, \ldots, n$, by $(24) u_{i}^{k-h_{j}} \geq u_{i}^{k-h_{j}+1} \geq \ldots \geq u_{i}^{k-h_{j+1}-1}$ for $i=$ $1, \ldots, n$ where the superscript indices are taken modulo $k$, and $h_{n+1}=h_{1}$. This implies

$$
\sum_{i=1}^{n} u_{i}^{k-h_{j}} \geq \sum_{i=1}^{n} u_{i}^{k-h_{j}+1} \geq \ldots \geq \sum_{i=1}^{n} u_{i}^{k-h_{j+1}-1}
$$

where the superscript indices are taken modulo $k$. Let $d=\sum_{i=1}^{n} p_{i}-c$. By (21), $\sum_{i=1}^{n} u_{i}^{t}=d$ for $t=0, \ldots, k-1$. Hence, by (27), for every $i, j=$ $1, \ldots, n$,

$$
u_{i}^{k-h_{j}}=u_{i}^{k-h_{j}+1}=\ldots=u_{i}^{k-h_{j+1}-1},
$$


where the superscript indices are taken modulo $k$.

Let $\Gamma$ be the directed graph on $n$ nodes defined as follows. For every $i, j \in\{1, \ldots, n\}, i \neq j$, there are $u_{i}^{k-h_{j}-1}-u_{i}^{k-h_{j}}$ arcs from $i$ to $j$ in $\Gamma$ (superscript indices being taken modulo $k$ ). By $(24), u_{i}^{k-h_{j}-1}-u_{i}^{k-h_{j}} \geq 0$. Notice that, since $\sum_{i=1}^{n} u_{i}^{t}=d$ for $t=0, \ldots, k-1$, then, given $j \in\{1, \ldots, n\}$, $\sum_{i=1}^{n} u_{i}^{k-h_{j}-1}-\sum_{i=1}^{n} u_{i}^{k-h_{j}}=0$, so

$$
\sum_{\substack{i=1 \\ i \neq j}}^{n}\left(u_{i}^{k-h_{j}-1}-u_{i}^{k-h_{j}}\right)=u_{j}^{k-h_{j}}-u_{j}^{k-h_{j}-1} .
$$

Notice that the value of the left-hand-side of the latter expression is the number of arcs in $\Gamma$ entering $j$. On the other hand, one can readily verify that, by (28), $u_{j}^{k-h_{j}}-u_{j}^{k-h_{j}-1}=\sum_{i=1 i \neq j}^{n}\left(u_{j}^{k-h_{i}-1}-u_{j}^{k-h_{i}}\right)$, which is the number of arcs leaving $j$. Thus $\Gamma$ is Eulerian, i.e. for each node $j$ the number of arcs entering $j$ equals the number of arcs leaving $j$.

Therefore there exists a directed cycle $C$ in $\Gamma$. Let $(\bar{c}, \bar{p}, \bar{q}, \bar{v}, \bar{u}, \bar{w}, \bar{m})$ be the multipliers determining the inequality (26) associated with $C$. Let $v^{\prime}=v-\bar{v}, u^{\prime}=u-\bar{u}, w^{\prime}=w-\bar{w}, m^{\prime}=m-\bar{m}, c^{\prime}=c-\bar{c}, p^{\prime}=p-\bar{p}$, $q^{\prime}=q-\bar{q}$. Then $\left(c^{\prime}, p^{\prime}, q^{\prime}, v^{\prime}, u^{\prime}, w^{\prime}, m^{\prime}\right)$ satisfy $(21)$, and $(c, p, q, v, u, w, m)=$ $(\bar{c}, \bar{p}, \bar{q}, \bar{v}, \bar{u}, \bar{w}, \bar{m})+\left(c^{\prime}, p^{\prime}, q^{\prime}, v^{\prime}, u^{\prime}, w^{\prime}, m^{\prime}\right)$, therefore $(c, p, q, v, u, w, m)$ does not determine a facet-defining inequality unless $\Gamma$ is just a cycle.

Remark 24 If a cycle inequality (14) associated with a cycle $C$ of $D$ and partition $(X, T)$ is facet-defining for $\operatorname{conv}\left(X^{C M F}\right)$, then $f_{i} \neq f_{j}$ for every $i, j \in V(C), i \neq j$.

Proof. Suppose there exist $h, k \in V(C)$ with $h \neq k$ and $f_{h}=f_{k}$. Among such $h, k$, choose two minimizing $|h-k|$. Since $f_{1} \geq f_{2} \geq \ldots \geq f_{n}$, the above choice implies that, for every $i \in V(C)$, either $i \geq h, k$ or $i \leq h, k$.

If $C$ has length two, then the corresponding cycle inequality is implied by the original inequalities. So we assume the $C$ has length at least three. Let $h^{\prime}$ be the predecessor of $h \in C$. Since $C$ has length at least three, we may assume $h^{\prime} \neq k$. Let $P^{\prime}$ be the unique directed path in $C$ from $k$ to $h^{\prime}$, and $C^{\prime}$ be the cycle $k, P^{\prime}, h^{\prime}, k$. By the choice of $h, k$, we have that $h^{\prime}>h$ if and only if $h^{\prime}>k$, and, obviously, $f_{h^{\prime}}-f_{h}=f_{h^{\prime}}-f_{k}$.

Let $k^{\prime}$ be the predecessor of $k \in C$. We consider two cases: $k^{\prime} \neq h$, or $k^{\prime}=h($ in which case $(h, k) \in C)$.

If $k^{\prime} \neq h$, let $P^{\prime \prime}$ be the unique directed path in $C$ from $h$ to $k^{\prime}$, and $C^{\prime \prime}$ be the cycle $h, P^{\prime \prime}, k^{\prime}, h$. Again, by the choice of $h, k$, we have that $k^{\prime}>k$ if and only if $k^{\prime}>h$, and $f_{k^{\prime}}-f_{k}=f_{k^{\prime}}-f_{h}$. One can now verify that the cycle inequality defined by $C$ and $(X, T)$ is the sum of the cycle inequalities defined by $C^{\prime}$ and $(X, T)$, and $C^{\prime \prime}$ and $(X, T)$. 
If $(h, k) \in C$, we have four cases. The cycle inequality defined by $C$ and $(X, T)$ is the sum of the cycle inequalities defined by $C^{\prime}$ and $(X, T)$ and

- $s+r_{h}+x_{h} \geq b_{h}$ if $h>k, h \in X$;

- $s+r_{h}+z_{h} \geq b_{h}$ if $h>k, h \in T$;

- $r_{h} \geq 0$ if $h<k, h \in X$;

- $r_{h}+z_{h} \geq 0$ if $h<k, h \in T$.

Example. Consider an instance of the continuous mixing set with flows with $n=4, b_{1}=5 / 6, b_{2}=9 / 6, b_{3}=20 / 6, b_{4}=1 / 6$. Thus $k=6, h_{1}=5$, $h_{2}=3, h_{3}=2, h_{4}=1$. Let $X=\{1,3\}, T=\{2,4\}$. The graph on the left in Figure 6 depicts the extended formulation for $\operatorname{conv}\left(S^{(X, T)}\right)$.

Consider the directed cycle $C=(1,4),(4,2),(2,3),(3,1)$. The graph on the right in Figure 6 depicts the multipliers $(\bar{c}, \bar{p}, \bar{q}, \bar{v}, \bar{u}, \bar{w}, \bar{m})$ defined in the proof of Theorem 23 that determine inequality (26). One obtains

$$
-12 s+6 \sum_{i=1}^{4} y_{i}+6 z_{2}^{\prime}+6 z_{4}^{\prime}+4 x_{1}^{\prime}+3 x_{3}^{\prime}+5 \ell_{2}+2 \ell_{4} \geq 4 .
$$

Dividing by 6 and substituting $y_{i}=s+r_{i}, i=1,2,3,4, z_{1}^{\prime}=z_{1}-5 / 6$, $z_{2}^{\prime}=z_{2}-9 / 6, z_{3}^{\prime}=z_{3}-20 / 6, z_{4}^{\prime}=z_{4}^{\prime}-1 / 6, x_{1}^{\prime}=x_{1}, x_{2}^{\prime}=x_{2}-1$, $x_{3}^{\prime}=x_{3}-3, x_{4}^{\prime}=x_{4}, \ell_{1}=0, \ell_{2}=1, \ell_{3}=3, \ell_{4}=0$, one obtains the cycle inequality associated with $C$

$$
2 s+\sum_{i=1}^{4} r_{i}+z_{2}+z_{4}+\frac{4}{6} x_{1}+\frac{3}{6} x_{3} \geq \frac{18}{6} .
$$

Continuous mixing set The continuous mixing set, studied by Van Vyve [13], is the following:

$$
X^{C M}=\left\{(s, r, x) \in \mathbb{R} \times \mathbb{R}_{+}^{n} \times \mathbb{Z}^{n} \mid s+r_{i}+x_{i} \geq b_{i}, i=1, \ldots, n\right\} .
$$

Next we state Van Vyve's result and show how to derive it from Theorem 23.

Theorem 25 (Van Vyve [13]) $\operatorname{conv}\left(X^{C M}\right)$ is defined by the inequalities $s+r_{i}+x_{i} \geq b_{i}, r_{i} \geq 0, i=1, \ldots, n$, and by the inequalities

$$
\begin{gathered}
\gamma_{C} s+\sum_{i \in V(C)} r_{i}+\sum_{\substack{(i, j) \in C \\
i<j}}\left(f_{i}-f_{j}\right) x_{i}+\sum_{\substack{(i, j) \in C \\
i>j}}\left(1+f_{i}-f_{j}\right) x_{i} \geq \\
\sum_{\substack{(i, j) \in C \\
i>j}} f_{j}+\sum_{\substack{(i, j) \in C \\
i<j}}\left(f_{i}-f_{j}\right)\left\lfloor b_{i}\right\rfloor+\sum_{\substack{(i, j) \in C \\
i>j}}\left(1+f_{i}-f_{j}\right)\left\lfloor b_{i}\right\rfloor
\end{gathered}
$$

for every directed cycle $C$ of $D$. 

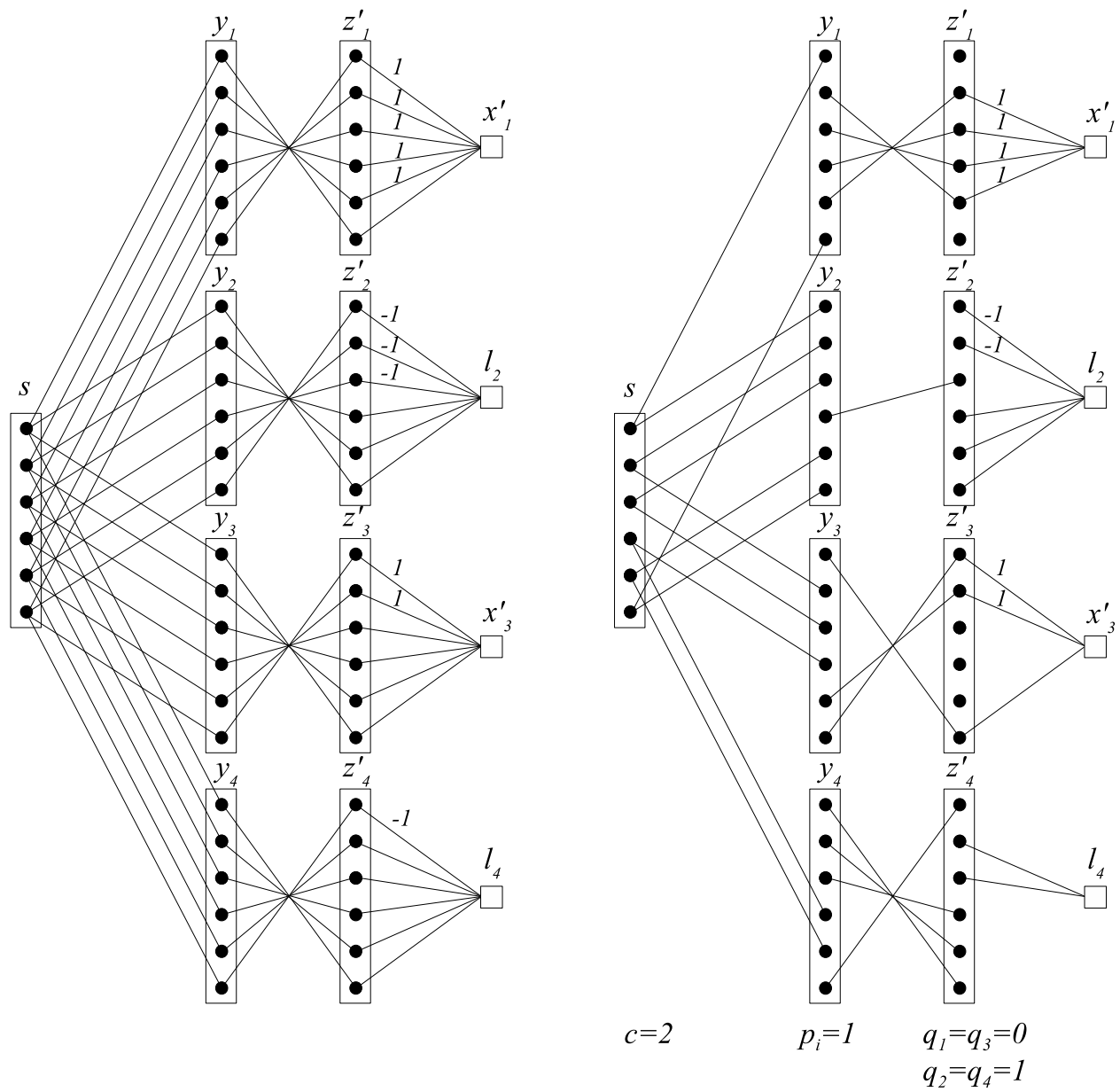

Figure 6: Extended formulation for $\operatorname{conv}\left(S^{(X, T)}\right)$ in the example. The black dots represent the additional variables associated to each of the variables $s$, $y_{i}, z_{i}^{\prime}, x_{i}^{\prime}, \ell_{i}$. The indices $t$ go from 0 on top to $k-1$ on the bottom of each stack. The \pm 1 s on the edges represent the non-zero right-hand-sides of the corresponding inequalities. 
Proof. Let $X=\{1, \ldots, n\}$ and $T=\emptyset$. Note that a point $(s, r, x)$ is in $\operatorname{conv}\left(X^{C M}\right)$ if and only if the point $(s, r, x, x)$ is in $\operatorname{conv}\left(X^{(X, T)}\right) \cap\{(s, r, z, x \mid z=$ $x\}$. Since the latter polyhedron is a face of $\operatorname{conv}\left(X^{(X, T)}\right)$, we have that any facet-defining inequality for $\operatorname{conv}\left(X^{C M}\right)$ is obtained from some facet-defining inequality for $\operatorname{conv}\left(X^{(X, T)}\right)$ by replacing $z_{i}$ by $x_{i}, i=1, \ldots, n$. Given a directed cycle $C$ of $D$, if we substitute $z_{i}$ with $x_{i}, i=1, \ldots, n$, in the cycle inequality (14) relative to $(X, T)$ and $C$, we obtain precisely (29).

Description of $X^{C M F_{+}}$Conforti, Di Summa, and Wolsey [3], give a polynomial size extended formulation for the set $X_{n}^{C M F_{+}}=X_{n}^{C M F} \cap\{(s, r, z, x) \mid s \geq$ $0\}$.

Note that in [3], the authors used the name "continuous mixing set with flows" for the set $X_{n}^{C M F_{+}}$. As demonstrated for the mixing set above, it is easy to see that $X_{n}^{C M F_{+}}$can be considered as a face of $X_{n+1}^{C M F}$. Indeed, if we define $b_{n+1}=0$, then a point $(s, r, z, x)$ belongs to $\operatorname{conv}\left(X_{n}^{C M F_{+}}\right)$if and only if $(s, r, 0, z, 0, x, 0)$ belongs to

$$
\operatorname{conv}\left(X_{n+1}^{C M F}\right) \cap\left\{\left(s, r, r_{n+1}, z, z_{n+1}, x, x_{n+1}\right) \mid r_{n+1}=0, z_{n+1}=0, z_{n+1}=x_{n+1}\right\} .
$$

Since the latter polyhedron is a face of $\operatorname{conv}\left(X_{n}^{C M F}\right)$, any facet-defining inequality for $\operatorname{conv}\left(X_{n}^{C M F_{+}}\right)$is obtained from some facet-defining inequality for $\operatorname{conv}\left(X_{n+1}^{C M F}\right)$ by replacing $r_{n+1}, z_{n+1}$ and $x_{n+1}$ with 0 .

Separating cycle inequalities for $X^{C M F}$ We conclude the section by discussing how to separate the cycle inequalities (14) for the continuous mixing set with flows. The polynomial algorithm we provide is almost identical to that proposed by Van Vyve [13] for the continuous mixing set.

We construct a digraph $D^{\prime}$ on the node set $\{1, \ldots, n\}$, where for each pair $\{i, j\} \subseteq\{1, \ldots, n\}, i \neq j$, there are two parallel arcs $e_{i j}^{X}$ and $e_{i j}^{T}$ from $i$ to $j$ and two parallel arcs $e_{j i}^{X}$ and $e_{j i}^{T}$ from $j$ to $i$. Given a point $(\bar{s}, \bar{r}, \bar{z}, \bar{x})$, we assign $\operatorname{costs} c_{e}$ to the arcs $e$ of $D^{\prime}$ as follows.

$$
\begin{array}{rlrl}
c_{e_{i j}^{X}}=\bar{r}_{i}+\left(f_{i}-f_{j}\right) \bar{x}_{i}-\left(f_{i}-f_{j}\right)\left\lfloor b_{i}\right\rfloor, & & i<j ; \\
c_{e_{i j}^{T}}=\bar{r}_{i}+\bar{z}_{i}-\left(f_{i}-f_{j}\right)\left\lfloor b_{i}\right\rfloor, & & i<j ; \\
c_{e_{i j}^{X}}=\bar{s}+\bar{r}_{i}+\left(1+f_{i}-f_{j}\right) \bar{x}_{i}-f_{j}-\left(1+f_{i}-f_{j}\right)\left\lfloor b_{i}\right\rfloor, & i>j ; \\
c_{e_{i j}^{T}}=\bar{s}+\bar{r}_{i}+\bar{z}_{i}-f_{j}-\left(1+f_{i}-f_{j}\right)\left\lfloor b_{i}\right\rfloor, & & i>j .
\end{array}
$$

Compute a directed cycle $C$ in $D^{\prime}$ of minimum mean cost. This can be done in polynomial time [7]. If $C$ has negative cost, let $X$ be the set of nodes $i \in V(C)$ such that the unique arc leaving $i$ in $C$ is $c_{e_{i j}^{X}}$, for some $j \in V(C)$, and let $T$ be the set of nodes $i \in V(C)$ such that the unique arc leaving $i$ in $C$ is $c_{e_{i j}^{T}}$, for some $j \in V(C)$. Then $(\bar{s}, \bar{r}, \bar{z}, \bar{x})$ violates the cycle 
inequality (14) defined by $C, X, T$. On the other hand, if $C$ has non-negative cost, then no cycle inequality is violated by $(\bar{s}, \bar{r}, \bar{z}, \bar{x})$.

\section{Final remarks}

When $G$ is a tree, several properties of the facet-defining inequalities for $\operatorname{conv}\left(S^{(G, I)}\right)$ have been given in Section 4.1. However, a full characterization of such inequalities is known only for special cases. Here we propose the following conjecture.

Conjecture 26 Let $G$ be a tree and $I$ be the leaves of $G$. Let $(c, u)$ be a vector satisfying (6) and $i \in I$ be a leaf of $G$. If $\pi^{(c, u)} x \geq \delta^{(c, u)}$ is facetdefining for $S^{(G, I)}$, then $u_{n(i) i}^{t} \in\{0,1\}, t=0, \ldots, k-1$.

By Lemma 19, Conjecture 26 would imply that, for any $i \in I$, the vector $u_{n(i) i}$ is a 0,1 vector where the 1 entries are consecutive starting from

entry $u_{n(i) i}^{k-h_{i}}$. Furthermore, Remark 20 implies that all entries of $(c, u)$ are determined by the vectors $u_{n(i) i}$ for $i \in I$.

The following example shows that the vector $u$ of multipliers is not a 0,1 vector in general, even if $G$ is a tree.

Example. Consider the set $S^{(G, I)}$ defined by the following

$$
\begin{array}{rlrl}
x_{1}+x_{6} & \geq 2 / 5 & x_{11}+x_{12} & \geq 0 \\
x_{2}+x_{7} \geq 3 / 5 & x_{12}+x_{9} & \geq 0 \\
x_{3}+x_{8} & \geq 4 / 5 & x_{12}+x_{10} & \geq 0 \\
x_{6}+x_{11} & \geq 0 & x_{9}+x_{4} \geq 0 \\
x_{7}+x_{11} \geq 0 & x_{10}+x_{5} \geq 4 / 5 \\
x_{8}+x_{11} \geq 0 & I=\{1,2,3,4,5\} .
\end{array}
$$

We checked with PORTA that the inequality

$2 x_{1}+2 x_{2}+2 x_{3}+3 x_{4}+3 x_{5}+5 x_{6}+5 x_{7}+5 x_{8}+5 x_{9}+5 x_{10}+15 x_{11}+10 x_{12} \geq 9$

is facet-defining for $\operatorname{conv}\left(S^{(G, I)}\right)$. It can be verified that the unique multipliers $u$ that produce the above inequality are those depicted in Figure 7 . Two of the multipliers associated with the edge $\{11,12\}$, namely $u_{\{11,12\}}^{1}$ and $u_{\{11,12\}}^{2}$, have value 2 .

\section{References}

[1] T. Christof, A. Loebel, PORTA - Polyhedron Representation Transformation Algorithm, (c)1997-2009.

http://www.zib.de/Optimization/Software/Porta/ 


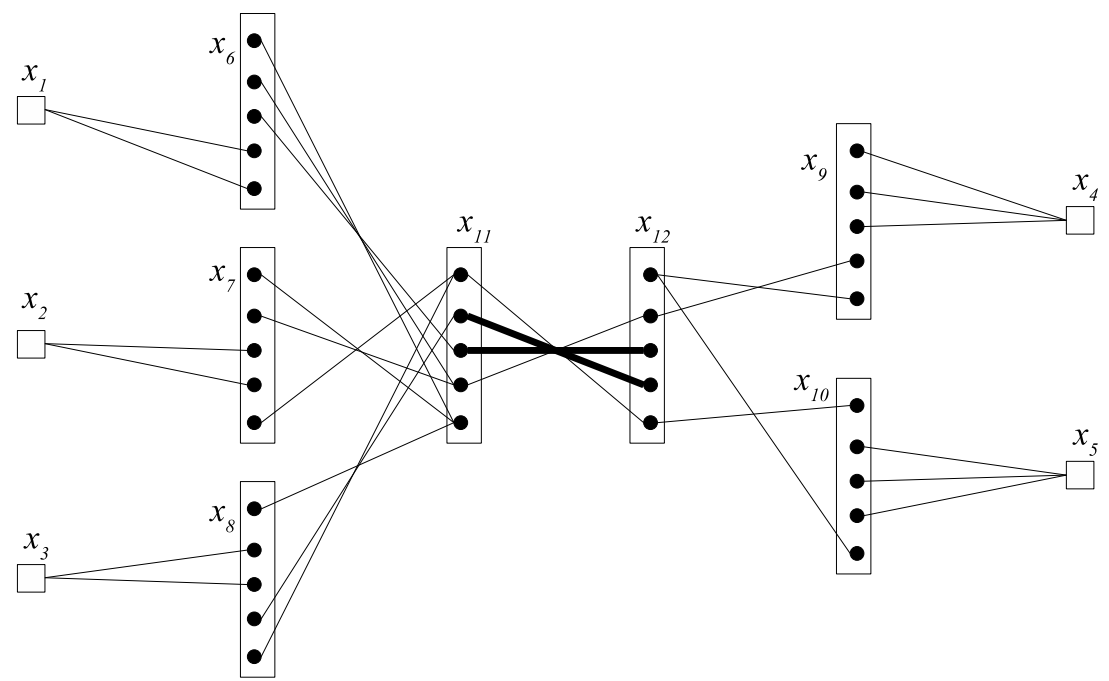

Figure 7: Regular edges represent multipliers with value 1, boldfaced edges represent multipliers with value 2 .

[2] M. Conforti, M. Di Summa, F. Eisenbrand, L.A. Wolsey, Network formulations of mixed-integer programs, Mathematics of Operations Research 34, 194-209 (2009).

[3] M. Conforti, M. Di Summa, and L. A. Wolsey, The intersection of continuous mixing polyhedra and the continuous mixing polyhedron with flows, in Proceedings of the 12th IPCO Conference, Lecture Notes in Computer Science LNCS 4513, Springer, (2007) 352-366.

[4] M. Conforti, B. Gerards and G. Zambelli, Mixed-integer vertex covers in bipartite graphs, in Proceedings of the 12th IPCO Conference, Lecture Notes in Computer Science LNCS 4513, Springer, (2007) 324-336.

[5] O. Günlük and Y. Pochet, Mixing mixed integer inequalities, Mathematical Programming 90 (2001), 429-457 .

[6] I. Heller and C.B. Tompkins, An extension of a theorem of Danzig's , in Linear Inequalities and Related Systems (H. W. Kuhn and A. W. Tucker, eds.) Princeton University Press, Princeton, N.J., 1956, pp. 247-254.

[7] R. Karp, A characterization of the minimum cycle mean in a digraph, Discrete Mathematics 23 (1978), 309-311.

[8] A. Miller and L.A. Wolsey, Tight formulations for some simple MIPs and convex objective IPs, Mathematical Programming B 98 (2003), $73-88$. 
[9] G.L. Nemhauser, L.A. Wolsey, Integer and Combinatorial Optimization, Wiley Interscience, New York, 1988.

[10] Y. Pochet and L.A. Wolsey, Polyhedra for lot-sizing with Wagner-Whitin costs, Mathematical Programming 67 (1994), 297-324.

[11] Y. Pochet and L.A. Wolsey, Production Planning by Mixed Integer Programming, Springer Series in Operations Research and Financial Engineering, New York, 2006.

[12] A. Schrijver, Theory of Linear and Integer Programming, Wiley, New York, 1986.

[13] M. Van Vyve, The Continuous Mixing Polyhedron, Mathematics of Operations Research 30 (2005), 441-452. 